\title{
A level-set model for mass transfer in bubbly flows
}

\author{
Néstor Balcázar-Arciniega ${ }^{\mathrm{a}, \mathrm{b}, *}$, Oscar Antepara ${ }^{\mathrm{a}, \mathrm{b}}$, Joaquim Rigola ${ }^{\mathrm{a}}$, Assensi Oliva ${ }^{\mathrm{a}, *}$ \\ ${ }^{a}$ Heat and Mass Transfer Technological Center (CTTC), Universitat Politècnica de Catalunya - BarcelonaTech (UPC) ETSEIAT, Colom 11, 222 Terrassa, Barcelona, Spain ${ }^{1}$ \\ ${ }^{\mathrm{b}}$ Termo Fluids S.L., Avda Jacquard 97 1-E, 08222 Terrassa, Barcelona, Spain ${ }^{2}$
}

\section{A R T I C L E I N F O}

\section{Article history:}

Received 4 January 2019

Received in revised form 20 March 2019

Accepted 2 April 2019

Available online $\mathrm{xxxx}$

\section{Keywords:}

Bubbly flow

Heat transfer

Mass transfer

Unstructured meshes

Finite-volume method

Level-set method

Flux limiters

\begin{abstract}
A B S T R A C T
A level-set model is presented for simulating mass transfer or heat transfer in two-phase flows. The Navier-Stokes equations and mass transfer (or heat transfer) equation are discretized using a finite volume method on a collocated unstructured mesh, whereas a multiple marker level-set approach is used for interface capturing in bubble swarms. This method avoids the numerical coalescence of the fluid particles, whereas the mass conservation issue inherent to standard level-set methods is circumvented. Furthermore, unstructured flux-limiter schemes are used to discretize the convective term of momentum transport equation, level-set equations, and chemical species concentration equation, to avoid numerical oscillations around discontinuities, and to minimize the numerical diffusion. A convection-diffusionreaction equation is used as a mathematical model for the chemical species mass transfer at the continuous phase. Because the mathematical analogy between dilute mass transfer and heat transfer, the same numerical model is applicable to solve both phenomena. The capabilities of this model are proved for the diffusion of chemical species from a sphere, external mass transfer in the buoyancy-driven motion of single bubbles and bubble swarms. Results are extensively validated by comparison with analytical solutions and empirical correlations from the literature.
\end{abstract}

(c) 2019 Published by Elsevier Ltd.

\section{Introduction}

Bubbly flows are common in natural phenomena and industry [1]. Some applications can be found on steam generators of thermal power plants, microfluidic devices, and the so-called unit operations of the chemical engineering, e.g., bubble columns are used for separation processes, or as chemical and biochemical reactors [2-4]. In these operations, bubbles or droplets are typically injected at the bottom of the column into a liquid phase, while a gas diffuses and reacts with dissolved reactants. Although some empirical correlations have been proposed for estimation of heat and mass transfer rates from bubbles or droplets [5], there is a lack of detailed understanding of the complex interplay between fluid mechanics and mass transfer (or heat transfer). Since these small-scale phenomena affect the overall operation and control of multiphase systems, as well for future optimization and design,

\footnotetext{
${ }^{1}$ Web page: http://www.cttc.upc.edu/.

2 Web page: http://www.termofluids.com/.

* Corresponding authors at: Heat and Mass Transfer Technological Center (CTTC), Universitat Politècnica de Catalunya - BarcelonaTech (UPC) ETSEIAT, Colom 11, 222 Terrassa (Barcelona), Spain (N. Balcázar-Arciniega).

E-mail addresses: nestor@cttc.upc.edu (N. Balcázar-Arciniega),cttc@cttc.upc.edu (A. Oliva).
}

it is of great importance to improve the accuracy of their predictive models.

Bubbly flows with mass transfer or heat transfer, lead to a highly non-linear mathematical problem. Indeed, analytical methods are restricted to very special cases, whereas experiments can be difficult to perform due to limitations in optical access. On the other hand, the development of computer technology opens the possibility to use Direct Numerical Simulation (DNS) of the Navier-Stokes equations, as another approach to design noninvasive and controlled experiments of bubbly flows [6]. In this sense, multiple methods have been introduced in the last decades for DNS of interfacial flows, some examples include: level-set (LS) methods [7,8], conservative level-set (CLS) methods [9,10], volume-of-fluid (VOF) methods [11,12], coupled VOF/LS methods [13-16], and front tracking (FT) methods [17]. These approaches solve two-phase flow using the so-called one-fluid formulation [17], where the fluid interface is captured by means of a Eulerian approach (VOF, LS, CLS, VOF/LS) or a Lagrangian (FT) approach. Despite the fact that the idea behind these methods is similar, their numerical implementations may differ greatly.

In the context of the aforementioned methods, remarkable efforts have been done on the development of numerical models for heat transfer or mass transfer in two-phase flows. For instance, Davidson and Rudman [18], Bothe et al. [19], Alke et al. [20], Onea 


\section{UPCommons}

\section{Portal del coneixement obert de la UPC}

http://upcommons.upc.edu/e-prints

Aquesta és una còpia de la versió author's final draft d'un article publicat a la revista International Journal of Heat and Massa Transfer

http://hdl.handle.net/2117/167909

Article publicat / Published paper:

Balcázar-Arciniega, N., [et al.]. A level-set model for mass transfer in bubbly flows. International Journal of Heat and Mass Transfer, Agost 2019, vol. 138, p. 335-356. DOI:

$<$ 10.1016/j.ijheatmasstransfer.2019.04.008>.

(C) <2019>. Aquesta versió està disponible sota la llicència CC-BY- NCND 4.0 http://creativecommons.org/licenses/by-nc-nd/4.0/ 


\section{Nomenclature}

A

area vector

A interfacial area

$|a| \quad$ absolute value of $a$

$A L \quad$ Hinterland ratio

C concentration

$C_{\Gamma} \quad$ interface concentration

$d \quad$ distance function, differential

$d_{b} \quad$ bubble diameter

$\mathcal{D} \quad$ diffusivity

Da Damköler number

êunit vector

Eo Eötvös number

g gravity

$h \quad$ local grid-size

$H \quad$ Heaviside function

$\mathrm{Ha} \quad$ Hatta number

$k_{1} \quad$ first-order reaction constant

$k_{c} \quad$ mass transfer coefficient in the continuous phase

$l_{K o} \quad$ Kolmogorov length scale

$l_{B a} \quad$ Batchelor length scale

Mo Morton number

n interface unit normal

$N_{c v} \quad$ number of control volumes

$n_{d} \quad$ number of bubbles

$p \quad$ pressure

$\mathrm{Pe} \quad$ Peclet number

Re Reynolds number

$r \quad$ radial coordinate

$\dot{r} \quad$ chemical reaction rate

$S \quad$ surface

Sc Schmidt number

$t \quad$ time

$U_{T} \quad$ terminal drift velocity

$\mathbf{v} \quad$ velocity

$\overline{\mathbf{v}} \quad$ time-averaged velocity

$V \quad$ volume

We Weber number

$\mathbf{x}$ position

\begin{tabular}{ll}
$x, y, z$ & $\begin{array}{l}\text { cartesian coordinates } \\
\text { dot product }\end{array}$ \\
$\|\mathbf{v}\|$ & Euclidean norm $(\mathbf{v} \cdot \mathbf{v})^{1 / 2}$ \\
\multicolumn{3}{|c}{ Greek } & Symbols \\
$\nabla$ & gradient \\
$\Delta$ & increment \\
$\delta_{\Gamma}$ & Dirac delta function \\
$\rho$ & density \\
$\mu$ & viscosity \\
$\eta_{\rho}$ & density ratio \\
$\eta_{\mu}$ & viscosity ratio \\
$\phi$ & CLS function \\
$\tau$ & pseudo-time \\
$\varepsilon$ & re-initialization equation parameter \\
$\Gamma$ & interface \\
$\Omega$ & spatial domain \\
$\sigma$ & surface tension coefficient \\
$\kappa$ & interface curvature \\
$\omega_{z}$ & vorticity component in $z$
\end{tabular}

Subscripts

$f \quad$ face

$P \quad$ current cell

C, D, U,F cells

$d \quad$ dispersed phase

c continuous phase

$i \quad i$ th bubble

$p \quad$ projected

$x, y, z \quad$ cartesian components

$\infty \quad$ reference

\section{Superscripts}

$S \quad$ regularized

$0 \quad$ initial

* normalized et al. [21], Gupta et al. [22], Hayashi and Tomiyama [23] have introduced numerical models for simulating mass transfer of chemical species within and between fluids with deforming interfaces, using a VOF method. Bothe et al. [24] proposed a variable transformation of the species concentration, to include the concentration jump at the interface, into a single continuous variable. Bothe et al. [25], Francois and Carlson [26] have reported VOF-based methods for calculating reactive mass transfer, whereas a sub-grid-scale model for mass transfer in single rising bubbles at high Schmidt numbers has been introduced by Bothe et al. [27], Weiner and Bothe [28]. Further efforts in the context of the VOF method include the implementation of a Continuous Species Transfer (CST) model by Marshall et al. [29] and Deising et al. [30]. Yang and Mao [31], Wang et al. [32] introduced a transformation of concentrations, molecular diffusivity, mass transfer time and velocities, for simulating the conjugate inter-phase mass transfer in single bubbles or droplets, in the context of the level-set method. Ganguli and Kenig [33] proposed an approach to handle the concentration jump at the interface, that does not require a constant distribution coefficient. Darmana et al. [34] presented a model based on the fronttracking technique, which allows a priori computation of mass transfer coefficients for rising bubbles. Bhuvankar and Dabiri [35] presented a numerical study of the heat transfer improvement around a bubble rising near a wall in a shear flow by using a front-tracking method. Mao et al. [36], Figueroa-Espinoza and Legendre [37] performed simulations using a body-conformal mesh around fixed-shape rising bubbles, whereas Arbitrary Lagrangian Eulerian (ALE) Interface-Tracking algorithm for reactive mass transfer in single bubbles has been reported by Falcone et al. [38].

DNS studies of heat transfer or mass transfer in bubble swarms are still quite limited. For instance, Dabiri and Tryggvason [39] studied the heat transfer in bubbly flows in a turbulent channel using the Front-Tracking method [17], furthermore this methodology was employed by Piedra et al. [40] to study the effect of the channel angle on the flow and the heat transfer. Deen and Kuipers [41] performed a numerical study of wall to liquid heat transfer in dispersed gas-liquid two-phase flow using VOF simulations, demonstrating that the heat transfer coefficient peaked near the bubbles. Aboulhasanzadeh et al. [42-44] developed a multiscale approach to compute the mass transfer from buoyant bubbles, using a boundary-layer approximation next to the bubble and a coarse grid for the rest of the flow, in the framework of the Front-Tracking method [17]. Roghair et al. [45] presented an improved Front-Tracking technique for the simulation of mass transfer in dense bubbly flows, including first order chemical reactions in the continuous phase. Koynov et al. [46], Radl et al. [47] researched the impact of bubble swarm hydrodynamics on chem- 
ical reaction selectivities, using two-dimensional simulations and the front-tracking method [17].

Although previous efforts have introduced remarkable numerical findings, many other configurations and flow conditions are not explored yet. Since interface capturing methods lead to an artificial and potentially unphysical coalescence of bubbles, most of previous research about bubbly flows, with or without heat transfer or mass transfer, has been performed using the front-tracking method [17]. Moreover, the accuracy and capacity of novel numerical approaches to tackle heat or mass transfer in bubbly flows, e.g., the multiple marker CLS method [48], is still to be proven. Despite the fact that previous papers touched upon heat transfer or mass transfer in single bubbles or droplets using VOF, LS and FT methods, to the best of the authors' knowledge there are not previous studies in the context of the conservative level-set (CLS) method $[9,10]$. Indeed, this work aims to present a sufficiently general numerical methodology for simulating interfacial mass transfer processes (or heat transfer) in singles bubbles and bubble swarms, within the framework of the multiple marker CLS approach introduced in Balcázar et al. $[48,49]$. This approach includes the adoption of three-dimensional collocated unstructured meshes [10], as well as the possibility to activate adaptive mesh refinement [50]. Thus, using a finite-volume/CLS method [10], the accumulation of mass conservation error that is known to affect standard level-set formulations, is circumvented. Whereas the multiple marker approach $[48,49,51]$ avoids the numerical and potentially unphysical coalescence of fluid particles, taken into account bubble collisions in long-time simulations of bubbly flows. Finally, these numerical methods and algorithms are efficiently implemented in a parallel computational framework, which lead to an accurate and robust numerical tool for computing the Sherwood number in 3D bubbly flows, as a function of Reynolds number, Schmidt number, Damköler number and bubble volume fraction.

Further advantages of the unstructured finite-volume/CLS method [10], include the accurate computation of normals at the interface, employing a least-squares method based on a wide and symmetric nodes-stencil. Then, these normals are used for an accurate computation of the surface tension force, without reconstruction of distance functions as in coupled volume-of-fluid/level-set methods, and without explicit representation of the interface as in front-tracking methods. Since most computational operations are local; this method can be efficiently implemented on parallel computational platforms (see Appendix B). Furthermore, unstructured meshes are adaptable to complex domains, allowing for an efficient mesh distribution in regions where the interface resolution has to be maximized. On the other hand, unstructured fluxlimiter schemes designed to discretize convective terms of transport equations, avoid numerical oscillations around discontinuities, whereas the numerical diffusion is minimized. Finally, the finite-volume methodology is attractive due to the satisfaction of the integral forms of the conservation laws over the entire domain.

This paper is organized as follows: The mathematical formulation is reviewed in Section 2. Section 3 introduces the numerical methods. Model validation and numerical experiments, including the prediction of Sherwood number in single bubbles and bubble swarms, are presented in Section 4. Finally, concluding remarks and future work are discussed in Section 5.

\section{Mathematical formulation}

\subsection{Incompressible two-phase flow}

The Navier-Stokes equations for the dispersed fluid in $\Omega_{d}$ and continuous fluid in $\Omega_{c}$ can be combined into a set of equations in a global domain $\Omega=\Omega_{d} \cup \Omega_{c}$, with a singular source term for the surface tension force at the interface $\Gamma[17,10]$ :

$$
\begin{aligned}
\frac{\partial}{\partial t}(\rho \mathbf{v})+\nabla \cdot(\rho \mathbf{v v})= & -\nabla p+\nabla \cdot \mu\left(\nabla \mathbf{v}+(\nabla \mathbf{v})^{T}\right) \\
& +\left(\rho-\rho_{0}\right) \mathbf{g}+\mathbf{f}_{\sigma}
\end{aligned}
$$

$\nabla \cdot \mathbf{v}=0$

where $\mathbf{v}$ and $p$ denote the fluid velocity and pressure field respectively, $\rho$ is the fluid density, $\mu$ is the dynamic viscosity, $\mathbf{g}$ is the gravitational acceleration, $\mathbf{f}_{\sigma}$ is the surface tension force (Section 2.3), subscripts $d$ and $c$ denote the dispersed phase and continuous phase respectively. Since physical properties are assumed to be constant at each fluid-phase with a jump discontinuity at the interface, these can be written as:

$\rho=\rho_{c} H_{c}+\rho_{d} H_{d}, \mu=\mu_{c} H_{c}+\mu_{d} H_{d}$,

where $H_{c}$ is the Heaviside step function that is one in $\Omega_{c}$ and zero elsewhere, and $H_{d}=1-H_{c}$. At discretized level a continuous treatment of physical properties is adopted in order to avoid numerical instabilities at the interface (Section 2.4). Furthermore, if periodic boundary condition is applied on the $y$-axis (aligned to $\mathbf{g}$ ), then a force $-\rho_{0} \mathbf{g}$ is added to the Navier-Stokes equations (Eq. (1)), with $\rho_{0}=V_{\Omega}^{-1} \int_{\Omega}\left(\rho_{d} H_{d}+\rho_{c} H_{c}\right) d V$, to prevent the acceleration of the entire flow field in the downward vertical direction due to the action of $\mathbf{g}[52,48,53]$. On the other hand, $\rho_{0}=0$ for simulations without periodic boundary conditions on the $y$-axis.

\subsection{Multiple marker CLS method}

There are three major challenges for simulating bubbly flows in the framework of interface capturing methods: the first is how to keep a sharp front of the fluid interface; the second is how to perform an efficient and accurate computation of surface tension forces; and the third is how to avoid the numerical merging of the bubbles. Regarding the first and second issues, this work adopts a finite-volume/CLS method introduced by Balcázar et al. [10] for interface capturing on general unstructured grids, whereas the third issue is addressed using a multiple marker CLS approach introduced in Balcázar et al. [48]. Thus, the present method employs different CLS functions (called markers), $\phi_{1}, \ldots, \phi_{n_{d}}$, to follow the motion of each fluid-particle $\left(\Omega_{i}\right)$ contained by the dispersed phase $\left(\Omega_{d}\right)$. As a consequence, two or more CLS functions can be captured in the same control volume, allowing for the collision of the fluid particles without numerical merging of their interfaces, as illustrated in Fig. 1.

In the CLS method the interface is implicitly represented by the 0.5 iso-surface of the regularized indicator function $\phi_{i}$ :

$\phi_{i}(\mathbf{x}, t)=\frac{1}{2}\left(\tanh \left(\frac{d_{i}(\mathbf{x}, t)}{2 \varepsilon}\right)+1\right) \quad, i=1, \ldots, n_{d}$,

where $d_{i}$ is the signed distance function associated to the ith fluid particle, and $\varepsilon$ a parameter that sets the thickness of the CLS profile [10]. Eq. (4) is used to initialize the CLS functions $\left(\phi_{i}\right)$, from the distance functions $\left(d_{i}\right)$ generated for each fluid particle. Since the velocity field is solenoidal $(\nabla \cdot \mathbf{v}=0)$, the ith interface transport equation can be written in conservative form as follows:

$\frac{\partial \phi_{i}}{\partial t}+\nabla \cdot \phi_{i} \mathbf{v}=0 \quad, i=1,2, \ldots, n_{d}$.

Furthermore, an additional re-initialization equation is introduced to keep a sharp and constant interface profile:

$\frac{\partial \phi_{i}}{\partial \tau}+\nabla \cdot \phi_{i}\left(1-\phi_{i}\right) \mathbf{n}_{i}^{0}=\nabla \cdot \varepsilon \nabla \phi_{i}, \quad i=1,2, \ldots, n_{d}$. 


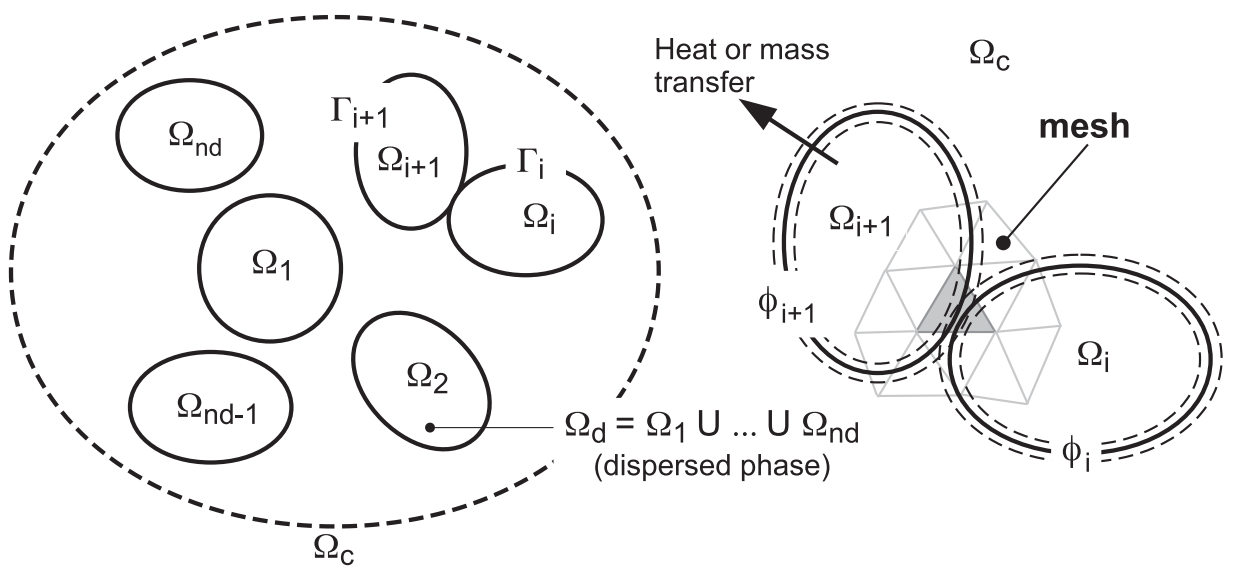

(continuous phase)

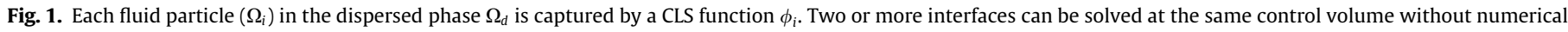
merging.

This equation is advanced in pseudo-time $\tau$, whereas $\mathbf{n}_{i}^{0}$ is the normal field at $\tau=0$. The compressive term in Eq. (6), $\phi_{i}\left(1-\phi_{i}\right) \mathbf{n}_{i}^{0}$, forces the CLS function to be compressed onto the interface along the normal vector $\mathbf{n}_{i}^{0}$. On the other hand, the diffusive term in Eq. (6), $\nabla \cdot \varepsilon \nabla \phi_{i}$, ensures the interface profile remains of characteristic thickness $\varepsilon=0.5 h^{0.9}$. The local grid size $(h)$ is measured as the average of the distances between the local cell centroid and the neighbors' cells centroids, around the faces of the local cell. Geometrical information on the interface $\Gamma_{i}$, such as normal vector $\mathbf{n}_{i}$ and curvature $\kappa_{i}$, are obtained as follows:

$\mathbf{n}_{i}\left(\phi_{i}\right)=\frac{\nabla \phi_{i}}{\left\|\nabla \phi_{i}\right\|}, \quad \kappa_{i}\left(\phi_{i}\right)=-\nabla \cdot \mathbf{n}_{i}\left(\phi_{i}\right)$.

The curvature is integrated over each finite-volume $\Omega_{P}$, to compute a cell averaged value:

$\kappa_{i, P}=-\frac{1}{V_{P}} \int_{\Omega_{P}} \nabla \cdot \mathbf{n}_{i} d V=-\frac{1}{V_{P}} \int_{S_{P}} \mathbf{n}_{i} \cdot d \mathbf{A}$,

where $\nabla \phi_{i}$ is calculated at each cell by means of the least-squares method applied to a wide and symmetric nodes-stencil based on the information of neighbor cells around vertices of the current cell $\Omega_{P}[10]$, and weighted by the inverse-distance between the current and neighbor cell-centroids [54], $\mathbf{A}$ is the area vector, $V_{P}$ is the volume of the current cell, $S_{P}$ is the surface of the current cell, and the subindex $P$ denotes the Pth control volume.

\subsection{Surface tension}

Implementing surface tension in a numerical scheme involves two issues: First, an accurate computation of the curvature $(\kappa)$ must be performed, and second, the resultant pressure jump must be applied appropriately to the fluids. Since the governing equations are discretized by means of a finite-volume approach, these issues are conveniently solved by means of the Continuous Surface Force (CSF) model introduced by Brackbill et al. [55]. This model has been extended to the multiple marker CLS method in $[48,49]$, as follows:

$\mathbf{f}_{\sigma}=\sum_{i=1}^{n_{d}} \sigma \kappa_{i}\left(\phi_{i}\right) \mathbf{n}_{i}\left(\phi_{i}\right) \delta_{\Gamma, i}^{s}=\sum_{i=1}^{n_{d}} \sigma \kappa_{i}\left(\phi_{i}\right) \mathbf{n}_{i}\left(\phi_{i}\right)\left\|\nabla \phi_{i}\right\|=\sum_{i=1}^{n_{d}} \sigma \kappa_{i}\left(\phi_{i}\right) \nabla \phi_{i}$,

where the index $n_{d}$ refers to the total number of fluid particles in the dispersed phase $\Omega_{d}$, and $\delta_{\Gamma, i}^{s}=\left\|\nabla \phi_{i}\right\|$ is the regularized Dirac delta function concentrated at the interface, as illustrated in
Fig. 2. Further discussions on the regularization of Dirac delta function for level-set methods can be found in [56]. Here, some properties of $\delta_{\Gamma, i}^{s}$ are remarked in the context of CLS method:

- At discretized level $\delta_{\Gamma, i}^{s}$ can be interpreted in the cell $\Omega_{P}$ as follows:

$$
\left(\delta_{\Gamma, i}^{s}\right)_{P}=\frac{1}{V_{P}} \int_{\Omega_{P}}\left(\delta_{\Gamma, i}^{s}\right) d V=\frac{\text { interfacial area in } \Omega_{P}}{V_{P}},
$$

where $V_{P}$ is the volume of $\Omega_{P}$. Therefore, the interfacial area of the ith bubble can be computed as $A_{i}=\int_{\Omega} \delta_{\Gamma, i}^{s} d V$.

- For any time: $\phi_{i}=\phi_{i}\left(d_{i}(\mathbf{x})\right)$ (Eq. (4)) and $\left\|\nabla d_{i}(\mathbf{x})\right\|=1$ because $d_{i}$ is a signed distance function [7,8], then $\delta_{\Gamma, i}^{s}=\left\|\nabla \phi_{i}\left(d_{i}(\mathbf{x})\right)\right\|=\left(\partial \phi_{i}\left(d_{i}\right) / \partial d_{i}\right)\left\|\nabla d_{i}(\mathbf{x})\right\|=\partial \phi_{i}\left(d_{i}\right) / \partial d_{i}$. Combination of the last result and Eq. (4) leads to $\delta_{\Gamma, i}^{s}=(4 \varepsilon)^{-1}\left(1-\left(\tanh \left(d_{i} /(2 \varepsilon)\right)\right)^{2}\right)$. The last function presents the property $\int_{-\infty}^{\infty} \delta_{\Gamma, i}^{s}\left(d_{i}\right) d d_{i}=1$, whereas $\delta_{\Gamma, i}^{s}$ has a peak at $d_{i}=0$ (interface) of $(4 \varepsilon)^{-1}$, as illustrated in Fig. 2. Since $\varepsilon=0.5 h^{a}$, then $\delta_{\Gamma, i}^{s}$ approach the analytical Dirac delta function as $h \rightarrow 0$.

Finite-volume discretization of the surface tension force is performed as follows: $\int_{\Omega_{p}} \mathbf{f}_{\sigma} d V=\left(\mathbf{f}_{\sigma}\right)_{P} V_{P}=\sum_{i=1}^{n_{d}} \sigma \kappa_{i, P}\left(\nabla \phi_{i}\right)_{P} V_{P}$ [10], with $\kappa_{i, P}$ evaluated by Eq. (8), and $\left(\nabla \phi_{i}\right)_{P}$ computed by means of the least-squares method.

\subsection{Regularization of physical properties}

In order to avoid numerical instabilities at the interface, fluid properties (Eq. (3)) are regularized using a global level-set function ( $\phi)[48,49,51]$ :

$\phi(\mathbf{x}, t)=\min \left\{\phi_{1}(\mathbf{x}, t), \ldots, \phi_{n_{d}-1}(\mathbf{x}, t), \phi_{n_{d}}(\mathbf{x}, t)\right\}$.

If smoothed Heaviside functions are defined as $H_{c}^{s}=\phi$ and $H_{d}^{s}=1-H_{c}^{s}$; then, physical properties are given by:

$\rho=\rho_{d} H_{d}^{s}+\rho_{c} H_{c}^{s}, \mu=\mu_{d} H_{d}^{s}+\mu_{c} H_{c}^{s}$.

In this research, $\phi_{i}$ is defined as $0.5 \leqslant \phi_{i} \leqslant 1$ in $\Omega_{c}$, and $0 \leqslant \phi_{i}<0.5$ in $\Omega_{d}$. Furthermore, $H_{d, i}^{s}=1-\phi_{i}$ denotes the smoothed Heaviside-step function for the ith bubble. On the other hand, if $\phi_{i}$ is defined as $0.5 \leqslant \phi_{i} \leqslant 1$ in $\Omega_{d}$, and $0 \leqslant \phi_{i}<0.5$ in $\Omega_{c}$, then $\phi=\max \left\{\phi_{1}, \ldots, \phi_{n_{d}}\right\}$ (Eq. (10)), whereas $H_{d}^{s}=\phi$ and $H_{d, i}^{s}=\phi_{i}$. 

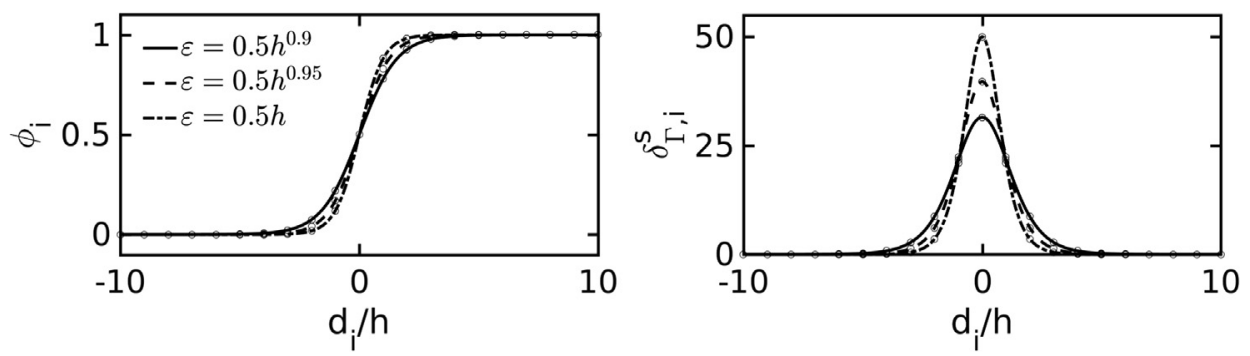

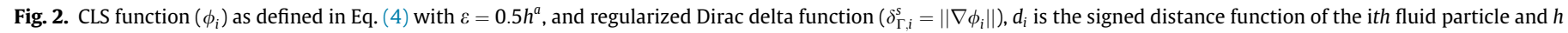
is the local grid size. The interface $\left(\Gamma_{i}\right)$ is located in $d_{i} / h=0$ which corresponds to $\phi_{i}=0.5$.

\subsection{Mass transfer}

For moderately soluble substances, the main resistance to mass transfer is located in the liquid phase $\left(\Omega_{c}\right)$ [5]; whereas the fluid inside the bubble is well mixed, such that the concentration in the bubbles can be considered as constant $[34,45,42]$. Furthermore, the present model involves that bubbles do not shrink and the concentration is not coupled to the hydrodynamics. As a consequence, only the solution outside the bubble is of interest. Taken into account these constraints, the mass balance of a chemical species is given by the following convection-diffusion-reaction equation defined in $\Omega_{c}$ :

$\frac{\partial C}{\partial t}+\nabla \cdot(\mathbf{v} C)=\nabla \cdot(\mathcal{D} \nabla C)+\dot{r}(C)$

where $C$ is the concentration of the chemical species, $\mathcal{D}$ is the diffusion coefficient or diffusivity which takes the value $\mathcal{D}_{c}$ in $\Omega_{c}$ and the value $\mathcal{D}_{d}$ in $\Omega_{d}$, whereas $\dot{r}(C)$ denotes the chemical reaction rate. In this work it is assumed a first order chemical reaction, then $\dot{r}(C)=-k_{1} C$, where $k_{1}$ is the reaction rate constant.

In this context, the concentration of the dissolved species $(C)$ at the centroids of the interface-cells (defined in next paragraph) is imposed by linear interpolation, using information of the concentration field from $\Omega_{c}$ (excluding the control volumes at the interface), and taken into account that the concentration at the bubble surface $\left(C_{\Gamma, c}\right)$ is constant. Thermodynamic equilibrium is assumed, therefore $C_{\Gamma, c}=H C_{\Gamma, d}$, where $H$ is the so-called Henry constant. The discontinuity introduced by Henry's law, if $H \neq 1$, can be treated by rescaling the concentration and diffusion coefficient as discussed by $[19,24,21,31]$, to obtain a continuous solution. As a consequence and without loss of generality, it is considered that the value of $C$ on the boundary of the bubble $\left(C_{\Gamma, c}\right)$ is to be given. Furthermore, this research aims to analyze steady-state mass transfer, which is only possible if the mass transfer resistance is located in $\Omega_{c}$. Cases with resistance in the droplet (internal mass transfer), or with resistance in both phases (conjugate mass transfer), will be analyzed in future works.

Given a cell $\Omega_{P}$ and its neighbor cells $\Omega_{i}$, then $\Omega_{P}$ is defined as an interface cell if there is at least one cell $\Omega_{k}$ for which $\phi_{P}>0.5$ and $\phi_{k}<0.5$, or $\phi_{P}<0.5$ and $\phi_{k}>0.5$, or if $\phi_{P}=0.5$ (see shaded cells in Fig. 3a). Here the subindex $P$ denotes the current cell $\Omega_{P}$, and subindex $k$ denotes the neighbor cells around the vertices of $\Omega_{P}$ (Cellslayer 1 in Fig. 3c). Once interface cells have been detected, the concentration at these cells $\left(C_{P}\right)$ is computed by linear interpolation, using a nodes-stencil $\left\{\mathbf{x}_{P}, \mathbf{x}_{\Gamma}, \mathbf{x}_{F_{p}}\right\}$, as illustrated in Fig. 3b. Where $\Gamma$ denotes the interface, $\mathbf{x}_{F_{p}}$ is the point projected from $\mathbf{x}_{F}$ to the line $l_{p}$, and $\mathbf{x}_{F} \in \Omega_{c}$ is the closest cell-centroid to the line $l_{p}$ selected from the cell-centroids around the vertices of $\Omega_{P}$, in a region of two cell layers around $\Omega_{P}$ (see Fig. $3 c$ ), $l_{p}$ is a line orthogonal to the interface which contains the points $\mathbf{x}_{P}$ and $\mathbf{x}_{\Gamma}$ (see Fig. $3 \mathrm{~b}$ ). Indeed, if $\Omega_{P}$ is an interface cell and $\mathbf{x}_{P} \in \Omega_{c}$, then $C_{P}$ is interpolated as follows:

$C_{P}=C_{\Gamma}-\frac{\left\|\mathbf{x}_{\Gamma}-\mathbf{x}_{P}\right\|}{\left\|\mathbf{x}_{\Gamma}-\mathbf{x}_{F_{p}}\right\|}\left(C_{\Gamma}-C_{F_{p}}\right)$.

$C_{F_{p}}$ can be approximated by a Taylor expansion as follows: $C_{F_{p}}=C_{F}+\left(\mathbf{x}_{F_{p}}-\mathbf{x}_{F}\right) \cdot\left(\nabla_{h} C\right)_{F}$. This operation is performed close to the interface-cells, where the resolution of the mesh is maximized. Furthermore, it is expected that $\mathbf{x}_{F_{p}} \approx \mathbf{x}_{F}$ as the mesh is refined, indeed $C_{F_{p}} \approx C_{F}$ is the approximation used in this work. The minimum distance from the point $\mathbf{x}_{F_{p}}$ to the interface $\Gamma$ is computed as follows:

|| $\mathbf{x}_{\Gamma}-\mathbf{x}_{F_{p}}\|=\| \mathbf{x}_{\Gamma}-\mathbf{x}_{P} \|+\left|\mathbf{n}_{P} \cdot\left(\mathbf{x}_{F}-\mathbf{x}_{P}\right)\right|$

with $\mathbf{n}_{P}=\left(\nabla_{h} \phi\right)_{P} /\left\|\left(\nabla_{h} \phi\right)_{P}\right\|$. The distance function $\left(\left|d\left(\mathbf{x}_{P}, t\right)\right|\right)$ at the cell-centroid $\mathbf{x}_{P}$ is approximated by means of Eq. (4), as follows:

$\left|d\left(\mathbf{x}_{P}, t\right)\right|=|| \mathbf{x}_{\Gamma}-\mathbf{x}_{P}||=\left|2 \varepsilon_{P} \tanh ^{-1}\left(2 \phi\left(\mathbf{x}_{P}, t\right)-1\right)\right|$.

While the present model is introduced for mass transfer, the mathematical analogy between heat transfer and mass transfer under the conditions of no dissipation, low mass flux and constant properties [5], permits to use this model for heat transfer processes.

\section{Numerical method}

The governing equations are solved with a finite-volume discretization of the physical domain on a collocated unstructured mesh, where scalar and vector variables $\left(p, \mathbf{v}, C, \ldots, \phi_{i}\right)$ are stored in the cell centroids, as first introduced in [10]. The convective term of momentum equation (Eq. (1)), chemical species mass transfer equations (Eq. (12)) and interface transport equation (Eq. (5)) is explicitly calculated approximating the fluxes at cell-faces by means of a TVD Superbee flux-limiter scheme [10] (see Eqs. (56)-(58) at Appendix A). Compressive term of the reinitialization equation (Eq. (6)) is approximated by using linear interpolation for the compressive flux at the cell-faces (Eqs. (56, $60)$, Appendix A). Diffusive terms of all discretized equations are centrally differenced (see DS1 in Table 4, Eqs. (61), (62) at Appen$\operatorname{dix} A$ ), whereas a distance-weighted linear interpolation is used to find the cell-face values unless otherwise stated. Gradients are computed at cell-centroids by using a least-squares method based on the information of the cell-nodes around the vertices of the current cell. The pressure-velocity coupling is solved by means of a standard fractional step projection method originally introduced by Chorin [57]. Hence, the global algorithm for simulation of mass transfer (or heat transfer) in two-phase flows is summarized as follows: 

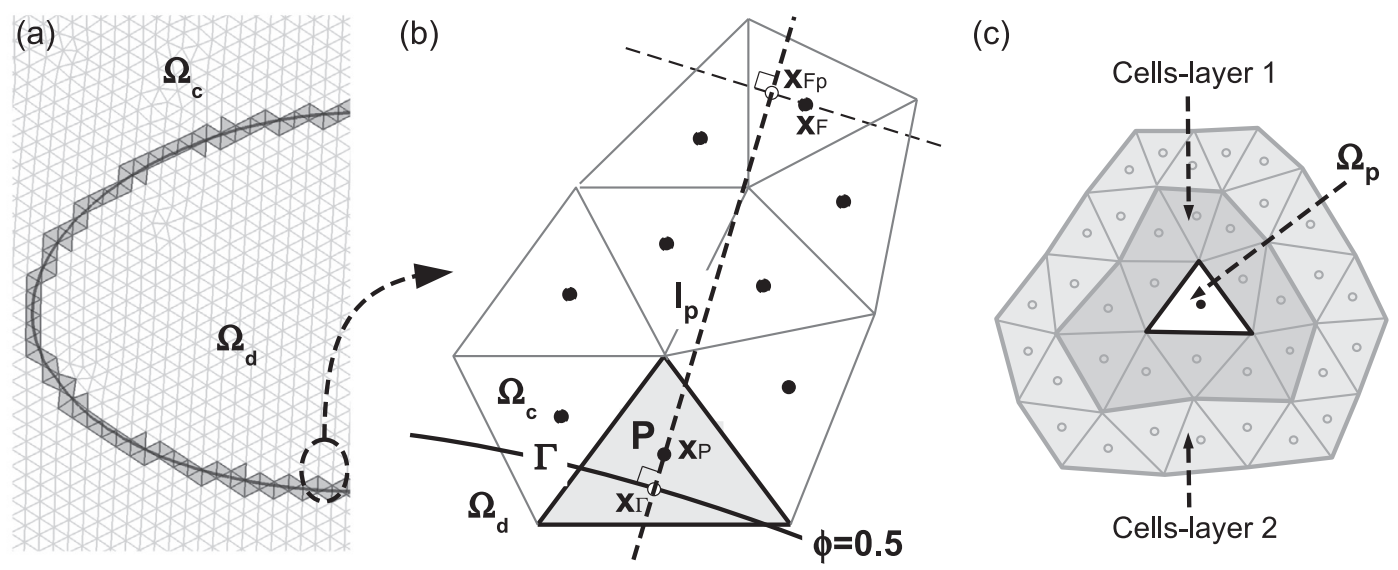

Fig. 3. (a) Interface-cells (shaded cells). (b) Nodes-stencil used to enforce a Dirichlet boundary condition at interface-cells. (c) Nodes-stencil around the current cell $\Omega_{p}$.

1. Initialize $\mathbf{v}\left(\mathbf{x}_{P}, 0\right), \phi_{i}\left(\mathbf{x}_{P}, 0\right), C\left(\mathbf{x}_{P}, 0\right)$ physical properties and interface geometric properties.

2. The time-step, $\Delta t$, which is limited by the CFL conditions and the stability condition for the capillary force [55], is calculated as follows:

$\Delta t=C_{t} \min \left(\frac{h}{\|\mathbf{v}\|}, \frac{\rho h^{2}}{\mu},\left(\frac{h}{\|\mathbf{g}\|}\right)^{1 / 2}, h^{3 / 2}\left(\frac{\rho_{1}+\rho_{2}}{4 \pi \sigma}\right)^{1 / 2}\right)$

where $C_{t}=0.1$ unless otherwise stated.

3. The fluid interfaces are advected using the multiple marker CLS method introduced by Balcázar et al. [48]. Indeed, advection equation (Eq. (5)) and re-initialization equation (Eq. (6)) are explicitly integrated in time with a 3-step third-order accurate TVD Runge-Kutta method [58]. Moreover, solving re-initialization equation (Eq. (6)) for the steady-state leads to a smooth transition of $\phi_{i}$ at the interface, proportional to the diffusion coefficient $\varepsilon=0.5 h^{0.9}$, where $h$ is the local grid size. This configuration corresponds to an interface thickness, with a distance between contours of $\phi=0.1$ and $\phi=0.9$, of around three times the grid size [10]. Furthermore, $\varepsilon$ is chosen as small as possible to keep a sharp representation of the interface, whereas the numerical stability of Eq. (6) is not affected. In present simulations two iterations per physical time step of reinitialization equation (Eq. (6)) are enough to maintain the profile of the CLS functions, whereas the time-step $(\Delta \tau)$ is restricted by the viscous term of Eq. (6) $[9,10]$ : $\Delta \tau=C_{\tau} \min \left\{\left(h^{2} / \varepsilon\right)_{P}\right\}, C_{\tau}=0.05$ and subindex $P$ denotes the Pth control volume.

4. Physical properties $(\rho, \mu)$ are updated at each control volume, according to the Section 2.4. Diffusivity is updated as follows: $\mathcal{D}=\mathcal{D}_{c}$ in $\Omega_{c}$, and $\mathcal{D}=\mathcal{D}_{d}$ in $\Omega_{d}$.

5. Mass transfer equation (Eq. (12)), is solved using an explicit Euler scheme on $\Omega_{c}$, excluding the interface cells:

$\frac{C-C^{n}}{\Delta t}=-\mathbf{C}_{C}^{n}+\mathbf{D}_{C}^{n}+\dot{r}(C)^{n}$

where $\mathbf{D}_{C}=\nabla_{h} \cdot\left(\mathcal{D} \nabla_{h} C\right)$ is the diffusion operator (see Appen$\operatorname{dix} A$, scheme DS1), $\mathbf{C}_{C}=\nabla_{h} \cdot(\mathbf{v} C)$ is the convective operator (see Appendix A), $\nabla_{h}$ is the gradient operator, whereas the super-index $n$ denotes the previous time step.

6. Concentration is computed at the interface cells by using Eq. (13).

7. A fractional-step projection method [57] is used to solve the pressure-velocity coupling. In the first step a predicted velocity $\left(\mathbf{v}^{*}\right)$ is computed at cells, as follows:

$$
\frac{\rho \mathbf{v}^{*}-\rho^{n} \mathbf{v}^{n}}{\Delta t}=-\mathbf{C}_{\mathbf{v}}^{n}+\mathbf{D}_{\mathbf{v}}^{n}+\left(\rho-\rho_{0}\right) \mathbf{g}+\sum_{i=1}^{n_{d}} \sigma \kappa_{i}\left(\phi_{i}\right) \nabla_{h} \phi_{i}
$$

where $\mathbf{D}_{\mathbf{v}}(\mathbf{v})=\nabla_{h} \cdot \mu \nabla_{h} \mathbf{v}+\nabla_{h} \cdot \mu\left(\nabla_{h} \mathbf{v}\right)^{T}$ is the diffusion operator, $\nabla_{h} \cdot \mu \nabla_{h} \mathbf{v}$ is approximated by a central difference scheme (see Appendix A, scheme DS1), $\nabla_{h} \cdot \mu\left(\nabla_{h} \mathbf{v}\right)^{T}$ is calculated by the Gauss-Theorem [10], $\nabla_{h} \phi_{i}$ and $\left(\nabla_{h} \mathbf{v}\right)^{T}$ are evaluated by using the least-squares method [10], $\kappa_{i}\left(\phi_{i}\right)$ is obtained at each cell according to Eq. (7), and $\mathbf{C}_{\mathbf{v}}(\rho \mathbf{v})=\nabla_{h} \cdot(\rho \mathbf{v v})$ is the convective operator (Superbee flux limiter scheme, Appendix A).

8. Application of the incompressibility constraint $\left(\nabla_{h} \cdot \mathbf{v}=0\right)$ into the corrector step (Eq. (20)) yields to a Poisson equation for the pressure field:

$\nabla_{h} \cdot\left(\frac{1}{\rho} \nabla_{h} p\right)=\frac{1}{\Delta t} \nabla_{h} \cdot\left(\mathbf{v}^{*}\right),\left.\mathbf{e}_{\partial \Omega} \cdot\left(\nabla_{h} p\right)\right|_{\partial \Omega}=0$.

Discretization of Eq. (19) leads to a linear system, which is solved by using a preconditioned conjugate gradient method. Here $\partial \Omega$ denotes the boundary of $\Omega$, excluding regions with periodic boundary condition, where information of the corresponding periodic nodes is used.

9. The predicted velocity ( $\left.\mathbf{v}^{*}\right)$ from Eq. (18), does not satisfy the incompressibility constraint. Therefore, a corrected velocity $(\mathbf{v})$ is obtained at cells, according to the fractional-step method [57]:

$\frac{\rho \mathbf{v}-\rho \mathbf{v}^{*}}{\Delta t}=-\nabla_{h}(p)$.

10. In order to avoid the pressure-velocity decoupling when the pressure projection is made on collocated meshes [59], and to fulfill the incompressibility constraint $\left(\nabla_{h} \cdot \mathbf{v}=0\right)$, a cell-face velocity $\left(\mathbf{v}_{f}\right)$ is interpolated as follows:

$\mathbf{v}_{f}=\sum_{q \in\{P, F\}} \frac{1}{2}\left(\mathbf{v}_{q}+\frac{\Delta t}{\rho_{q}}\left(\nabla_{h} p\right)_{q}\right)-\frac{\Delta t}{\rho_{f}}\left(\nabla_{h} p\right)_{f}$

where $P$ and $F$ denote the adjacent cell nodes with a common face $f$, and $\rho_{f}$ is computed by arithmetic average. The reader is referred to Appendix B of [49] for technical details on the origin of Eq. (21). This cell-face velocity is used to advect the CLS functions $\left(\phi_{i}\right)$ in Eq. (5), momentum $(\rho \mathbf{v})$ in Eq. (1), and chemical species concentration $(C)$ in Eq. (12).

11. Repeat steps $2-10$ until the desired simulation time. 
The numerical methods and algorithms introduced in this work have been implemented in the framework of the Computational Fluid Dynamics platform TermoFluids [60], which is an in-house code, based on C++ for object-oriented design, and Message Passing Interface (MPI) for parallel communications. The mesh is partitioned using ParMETIS library [61]. The parallel scalability of the computer code has been proven on the supercomputer MareNostrum IV, as detailed in Appendix B.

The reader is referred to [10,49,53] for additional technical details on the finite-volume discretization of the governing equations on unstructured meshes. Further verifications and validations of the CLS method for two-phase flows with surface tension, have been reported in our previous works [10,48,63,16,53]; whereas a comparison of CLS method [10] and coupled volume-of-fluid/ level-set method [16] is reported in [62].

\section{Model validation and numerical experiments}

The fluid dynamics of bubbles and droplets rising or falling freely in infinite media can be characterized by four dimensionless numbers [5]:

$M o=\frac{g \mu_{c}^{4} \Delta \rho}{\rho_{c}^{2} \sigma^{3}}, \quad$ Eo $=\frac{g d_{b}^{2} \Delta \rho}{\sigma}, \quad \eta_{\rho}=\frac{\rho_{c}}{\rho_{d}}, \quad \eta_{\mu}=\frac{\mu_{c}}{\mu_{d}}$,

where Mo is the Morton number, Eo is the Eötvös number, $\eta_{\rho}$ is the density ratio, $\eta_{\mu}$ is the viscosity ratio, $\Delta \rho=\left|\rho_{c}-\rho_{d}\right|, d_{b}$ is the spherical volume equivalent diameter of the bubble (or droplet). The subscripts $d$ and $c$ denote the dispersed fluid phase and the continuous fluid phase, respectively. The velocity of the ith bubble $\left(\mathbf{v}_{d, i}\right)$ is computed as follows:

$\mathbf{v}_{d, i}(t)=\frac{\int_{\Omega} \mathbf{v}(\mathbf{x}, t) H_{d, i}^{s}(\mathbf{x}, t) d V}{\int_{\Omega} H_{d, i}^{s}(\mathbf{x}, t) d V}$, for $i=1, \ldots, n_{d}$,

The spatial-averaged velocity in $\Omega$ and $\Omega_{d}$ are defined as:

$\mathbf{v}_{\Omega}(t)=V_{\Omega}^{-1} \int_{\Omega} \mathbf{v}(\mathbf{x}, t) d V, \mathbf{v}_{d}(t)=V_{d}^{-1} \int_{\Omega_{d}} \mathbf{v}(\mathbf{x}, t) d V$,

which are related to the continuous phase velocity $\left(\mathbf{v}_{c}(t)\right)$ by: $\mathbf{v}_{\Omega}(t)=(1-\alpha) \mathbf{v}_{c}(t)+\alpha \mathbf{v}_{d}(t)$, with $\quad \alpha=V_{d} / V_{\Omega}=\int_{\Omega_{d}} d V / \int_{\Omega} d V$ defined as the bubble fraction. In this work, the Reynolds number is expressed in terms of the drift velocity [52], $\Delta \mathbf{v}_{d, i}(t)=\mathbf{v}_{d, i}(t)-\mathbf{v}_{\Omega}(t)$, which corresponds to the bubble velocity with respect to a stationary container:

$\operatorname{Re}_{i}(t)=\frac{\rho_{c} d_{b}\left(\Delta \mathbf{v}_{d, i}(t) \cdot \hat{\mathbf{e}}_{y}\right)}{\mu_{c}}$, for $i=1, \ldots, n_{d}$

where $\hat{\mathbf{e}}_{y}$ is a unit vector parallel to $+y$ direction. The Reynolds number of the bubble swarm is computed as follows:

$\operatorname{Re}(t)=\frac{1}{n_{d}} \sum_{i=1}^{n_{d}} R_{i}(t)$

Furthermore, the terminal rise velocity $\left(\bar{U}_{T}\right)$ is computed as the time-averaged drift velocity when the steady state is achieved: $U_{T}=T^{-1} \int_{t_{0}}^{t_{0}+T} \Delta \mathbf{v}_{d, i}(t) \cdot \hat{\mathbf{e}}_{y} d t$. Once the velocity of each bubble is obtained by Eq. (23), bubble trajectories can be integrated as follows:

$\mathbf{x}_{d, i}(t)=\mathbf{x}_{d, i}^{0}+\int_{0}^{t} \mathbf{v}_{d, i}(t) d t$

where $\mathbf{x}_{d, i}^{0}$ is the initial position of the ith bubble centroid.

Large-scale models incorporate closures for mass transfer in the form of Sherwood number correlations $S h=f(R e, S c)$. Whereas, in bubble swarms it is expected an effect of the bubble fraction $[45,64]$. Thus, mass transfer (without chemical reaction) in bubbly flows can be characterized by the Sherwood number (Sh), and Schmidt number $(\mathrm{Sc})$ or Peclet number $(\mathrm{Pe})$ [5], defined in $\Omega_{c}$ as follows:

$S h=\frac{k_{c} d_{b}}{\mathcal{D}_{c}}, S c=\frac{\mu_{c}}{\rho_{c} \mathcal{D}_{c}}, P e=\frac{U_{T} d_{b}}{\mathcal{D}_{c}}=R e S c$,

where $k_{c}$ denotes the liquid-side $\left(\Omega_{c}\right)$ mass transfer coefficient. Dimensionless equations and boundary conditions for dilutesolution mass transfer and heat transfer, are the same [5]. Therefore, while results in this work are given in terms of Sh and Sc for mass transfer; the equivalent results for heat transfer can be obtained by replacing the Nusselt number $(\mathrm{Nu})$ by $\mathrm{Sh}$, and the Prandtl number $(\mathrm{Pr})$ by $\mathrm{Sc}$.

Finally, the Damköler (Da) number will be defined for mass transfer with homogeneous chemical reaction following firstorder kinetics, $\dot{r}(C)=-k_{1} C$, as follows:

$D a=\frac{k_{1} d_{b}^{2}}{\mathcal{D}_{c}}$.

This dimensionless number can be interpreted as the ratio of the reaction rate to the diffusion rate.

\subsection{Diffusion with chemical reaction of a stationary spherical bubble}

Validation of the numerical model for mass transfer with chemical reaction (Section 2.5) is performed by comparison of numerical results of diffusion from a stationary spherical bubble in a quiescent liquid, against the analytical solution. At steady state, the mass transfer of a chemical species with first order chemical reaction is given in spherical coordinates by:

$\frac{\mathcal{D}_{c}}{r^{2}} \frac{\partial}{\partial r}\left(r^{2} \frac{\partial C(r)}{\partial r}\right)-k_{1} C(r)=0$,

with boundary conditions $C\left(0.5 d_{b}\right)=C_{\Gamma, c}$, and $C(\infty)=0$. This equation has the following analytical solution:

$C(r)=C_{\Gamma, c} \frac{d_{b}}{2 r} \frac{e^{-a r}}{e^{-a d_{b} / 2}}$,

with $a=\left(k_{1} / \mathcal{D}_{c}\right)^{1 / 2}, C_{\Gamma, c}$ is the concentration at the interface from the side of $\Omega_{c}$, and $d_{b}$ is the bubble diameter.

In this simulation, the bubble of diameter $d_{b}$, is fixed at the center of a cubic domain $\Omega=\left[0,3.6 d_{b}\right] \times\left[0,3.6 d_{b}\right] \times\left[0,3.6 d_{b}\right]$. Furthermore $k_{1}=\{0.25,1\}, \mathcal{D}_{c}=5 \times 10^{-4}, C_{\Gamma, c}=1$ and $d_{b}=0.27 \overline{7}$. The velocity field is set to $\mathbf{v}=\mathbf{0}$ in $\Omega$, whereas the initial concentration is one in $\Omega_{d}$ and zero in $\Omega_{c}$. Neumann boundary condition is used on the confining boundary $\left(\partial_{\Omega}\right) . \Omega$ is discretized with tetrahedral control volumes, with averaged grid sizes $h=\left\{d_{b} / 36, d_{b} / 18, d_{b} / 9\right\}$ and number of control volumes $N_{c v}=\left\{29.5 \times 10^{6}, 3.6 \times 10^{6}, 0.5 \times 10^{6}\right\}$, respectively. A second mesh is set up using uniform hexahedral control volumes, with $h=\left\{d_{b} / 70, d_{b} / 42, d_{b} / 28\right\}$ and $N_{c v}=\left\{250^{3}, 150^{3}, 100^{3}\right\}$, respectively. Finally, the following error norms $\left(L_{1}, L_{2}\right)$ are defined for grid convergence studies:

$L_{1}=\frac{1}{N} \sum_{n=1}^{N}\left|x_{n, r e f}-x_{n}\right|, \quad L_{2}=\left(\frac{1}{N} \sum_{n=1}^{N}\left|x_{n, r e f}-x_{n}\right|^{2}\right)^{1 / 2}$,

where $x_{n}$ is the nth sample of quantity $x$, e.g. $x=C$, and $N$ is the number of samples. The analytical solution is taken as the reference solution $x_{n, \text { ref }}$.

Fig. 4a shows a comparison of numerical solution against analytical solution for the continuous radial concentration profile 



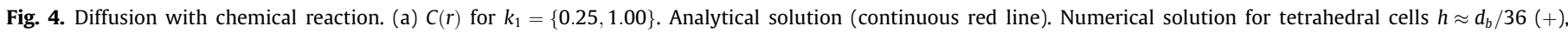


and $L_{2}(+)$ are defined in Eq. (32). (For interpretation of the references to colour in this figure legend, the reader is referred to the web version of this article.)

$(C(r))$, using tetrahedral cells and hexahedral control volumes. As can be observed numerical results for reaction rate constants $k_{1}=\{0.25,1\}$, are in close agreement with analytical solutions as the grid is refined. Fig. $4 \mathrm{~b}$ reports the order of convergence $(p)$, which is second order $(p \sim 2)$ for tetrahedral and hexahedral meshes. This verification demonstrates that the diffusion term and chemical reaction term have been correctly implemented on general unstructured meshes.

\subsection{Unsteady diffusion of a stationary spherical bubble}

This test case has been previously solved by Darmana et al. [34], in the framework of a front-tracking method and cartesian meshes. Here this problem is computed again to validate the proposed model for unsteady mass transfer. The species balance equation is written in spherical coordinates as follows:

$\frac{\partial C(r, t)}{\partial t}=\frac{\mathcal{D}_{c}}{r^{2}} \frac{\partial}{\partial r}\left(r^{2} \frac{\partial C(r, t)}{\partial r}\right)$
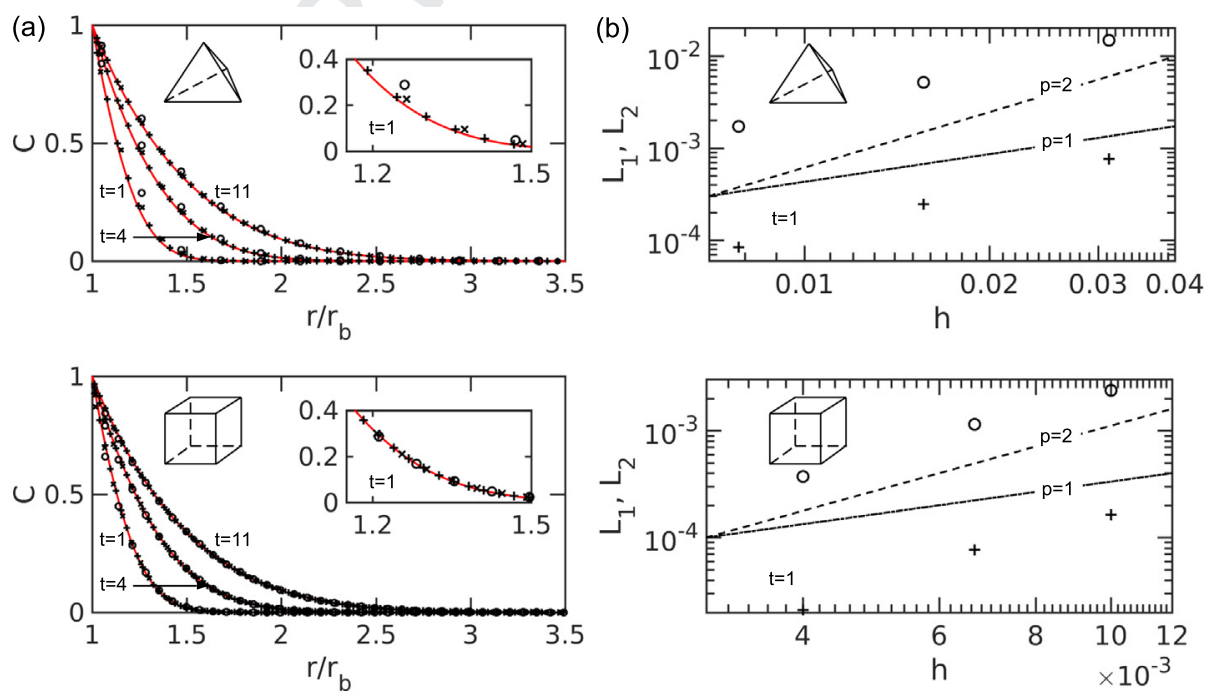

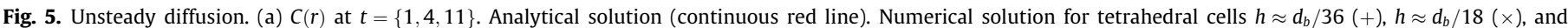

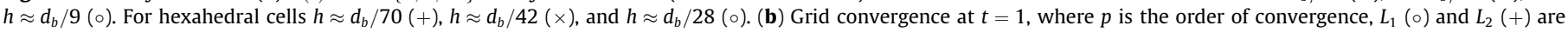
defined in Eq. (32). (For interpretation of the references to colour in this figure legend, the reader is referred to the web version of this article.) 
at $t=\{1,4,11\}$ converge to the analytical solutions as the grid is refined. Fig. $5 \mathrm{~b}$ shows the order of convergence $(p)$ at $t=1$, which is second order $(p \sim 2)$ for tetrahedral and hexahedral meshes. This verification demonstrates that the numerical code has been correctly implemented for unsteady mass transfer on general unstructured meshes.

\subsection{Mass transfer from single bubbles}

This section aims to perform an extensive validation of the level-set model, taken into account the interaction of hydrodynamics and external mass transfer in single rising bubbles. First, a grid convergence analysis as well as the assessment of diffusive schemes on unstructured meshes is carried out for hydrodynamics and mass transfer from a single bubble. Second, special attention is given to the discretization of convective terms on unstructured meshes, which is critical to conserve the numerical stability in bubbly flows with high-density ratio and high Reynolds number. Finally, a comparison of present numerical results for $\operatorname{Re}=\operatorname{Re}(E o, M o), C_{D}=C_{D}(E o, R e)$ and $S h=S h(R e, S c)$ against empirical correlations from the literature is performed.

In what follows, mass transfer coefficient $\left(k_{c}\right)$ from single rising bubbles is calculated using a mass balance of the chemical species in $\Omega_{c}$ :

$k_{c}(t)=\frac{V_{c}}{A\left(C_{\Gamma, c}-C_{\infty}\right)} \frac{d C_{c}}{d t}$,

where $C_{c}=V_{c}^{-1} \int_{\Omega_{c}} C d V, V_{c}$ is the volume of $\Omega_{c}, A=\int_{\Omega}\|\nabla \phi\| d V$ is the bubble surface, $C_{\Gamma, c}$ is the concentration at the interface from the liquid-side $\Omega_{c}$, and $C_{\infty}=0$ is the concentration far from the bubble interface.

\subsubsection{Mesh convergence analysis}

$\Omega$ is a cylindrical domain with height $L_{\Omega}$ and diameter $D_{\Omega}$ (see Table 1 ), where $d_{b}$ is the initial bubble diameter. $\Omega$ is discretized by triangular-prism cells, as illustrated in Fig. 6a, with the mesh concentrated around the y-axis, to maximize the grid resolution of the bubble. The local grid size $(h)$ on a region of diameter $2 d_{b}$ around the $\mathrm{y}$-axis is uniform (see Fig. 6b), with $h=\left\{d_{b} / 35, d_{b} / 30, d_{b} / 25\right\}$ (see meshes $M_{1}, M_{2}$ and $M_{3}$ in Table 1 ). As shown in Fig. $6 \mathrm{~b}$, the grid size grows exponentially from the concentrated region up to the lateral boundary, where it reaches a maximum size. These meshes were generated by a constant step extrusion of a two-dimensional unstructured grid along the y-axis, being the step size $L_{\Omega} / N_{p}=h$, where $N_{p}$ is the number of planes in which the $y$-axis is divided. Neumann boundary-condition is applied at the lateral, top and bottom walls. The initial bubble position is $(x, y, z)=\left(0,1.5 d_{b}, 0\right)$, on the $y$-axis, whereas both fluids are initially quiescent.

A first simulation is performed using dimensionless parameters $E o=3.125, M o=5 \times 10^{-6}, \eta_{\rho}=100, \eta_{\mu}=100, D a=0$ and $S c=1$. Fig. 7a and $b$ shows the time evolution of Reynolds number and Sherwood number for $h=\left\{d_{b} / 35, d_{b} / 30, d_{b} / 25\right\}$. Furthermore, an assessment of diffusive schemes with no-orthogonal correction is shown, by using black lines for the scheme DS1 (Table 4) and red lines for the scheme DS2 (Table 4). These schemes have been applied to all the transport equations, as described in Appendix A. It can be observed that simulations are not sensitive to the selected diffusive scheme, therefore the scheme DS1 (see Table 4) will be used throughout this research. Fig. 7c depicts vorticity contours $\left(\omega_{z}=\hat{\mathbf{e}}_{z} \cdot(\nabla \times \mathbf{v})\right)$ and concentration contours on the symmetry plane $x-y$ at $t^{*}=t \sqrt{g / d_{b}}=7.5$. It can be observed that numerical results for $h=d_{b} / 35$ and $h=d_{b} / 30$ are very close, whereas the grid convergence is demonstrated for hydrodynamics $\left(\omega_{z}\right)$ and mass transfer $(C)$. As a consequence, the grid size $h=d_{b} / 35$ is selected to perform further simulations unless otherwise stated.

As further physical argument to select the grid size, the socalled minimum-length scales are calculated. Following the work of Batchelor [66], the Kolmogorov $\left(l_{K o}\right)$ length scale describes the

Table 1

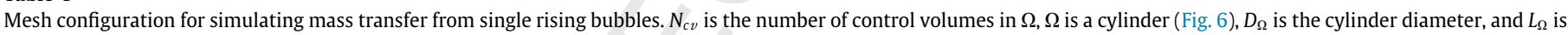
the cylinder height.

\begin{tabular}{|c|c|c|c|c|c|}
\hline Mesh & $N_{c v}$ & $h$ & Cell geometry & $D_{\Omega}$ & $L_{\Omega}$ \\
\hline M1 & $4.33 \times 10^{6}$ & $d_{b} / 35$ & Triangular-prism & $8 d_{b}$ & $10 d_{b}$ \\
\hline M2 & $3.65 \times 10^{6}$ & $d_{b} / 30$ & Triangular-prism & $8 d_{b}$ & $10 d_{b}$ \\
\hline M3 & $1.50 \times 10^{6}$ & $d_{b} / 25$ & Triangular-prism & $8 d_{b}$ & $10 d_{b}$ \\
\hline M4 & $7.17 \times 10^{6}$ & $d_{b} / 35$ & Hexahedral & $8 d_{b}$ & $16 d_{b}$ \\
\hline
\end{tabular}

(a)

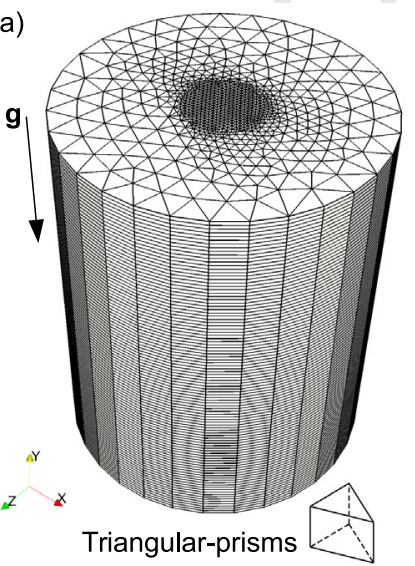

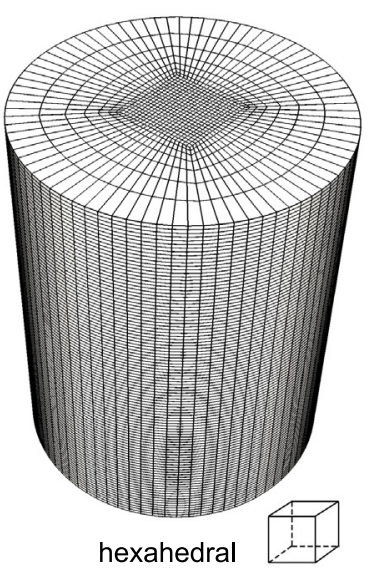

(b)

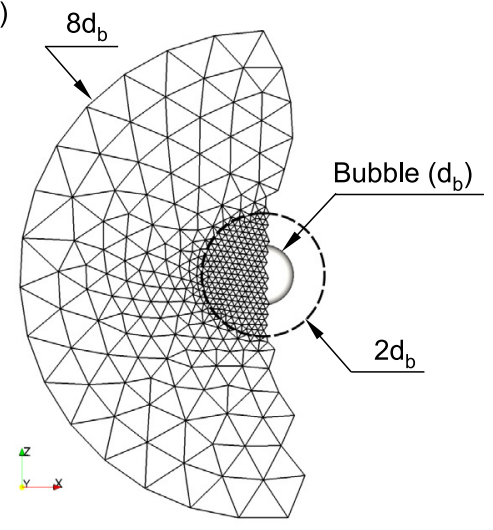

Fig. 6. Unstructured mesh configuration for mass transfer from single bubbles. (a) Meshes with hexahedral cells and triangular-prisms cells. (b) Cross-section ( $x-z)$. 

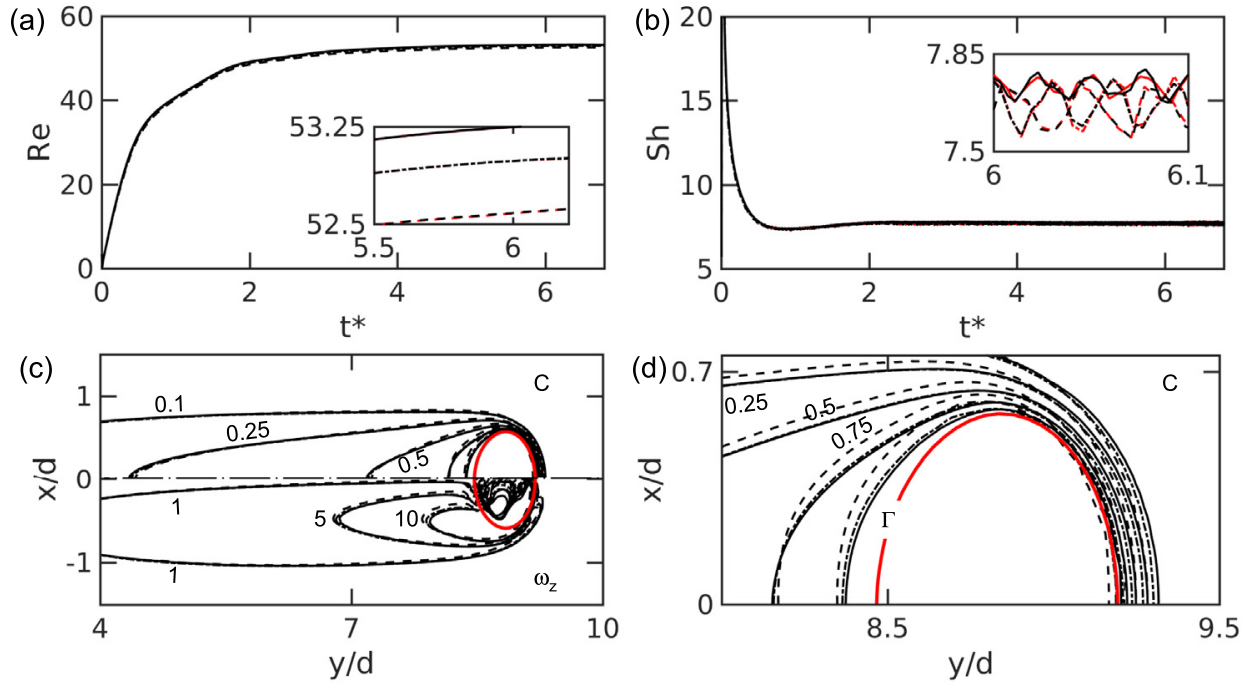

Fig. 7. $E o=3.125, M o=5 \times 10^{-6}, \eta_{\rho}=100, \eta_{\mu}=100, D a=0$ and $S c=1$. Grid convergence, $h=d_{b} / 35(-), h=d_{b} / 30(-\cdot), h=d_{b} / 25(--)$. Mesh composed by triangularprisms cells (Fig. 6a, Table 1). Diffusive schemes DS2 (red lines) and DS1 (black lines) as defined in Table 4. (a) Re(t) (Eq. (25)). (b) $\operatorname{Sh}(t)$ (Eq. (28)). (c) Concentration contours $C=\{0.1,0.5,0.75,0.9\}$, and vorticity contours $\omega_{z}=\hat{\mathbf{e}}_{z} \cdot \nabla \times \mathbf{v}=\{1,5,10\}$, at $t^{*}=t \sqrt{g / d_{b}}=7.5$, mesh $M_{1}$ (Table 1). (d) Zoom of concentration contours. (For interpretation of the references to colour in this figure legend, the reader is referred to the web version of this article.)

Table 2

Estimation of Kolmogorov $\left(l_{K o}\right)$ and Batchelor $\left(l_{B a}\right)$ length scales, where $d_{b}$ is the bubble diameter.

\begin{tabular}{|c|c|c|c|c|c|}
\hline Eo & Mo & $R e$ & Sc & $d_{b} / l_{K o}$ & $d_{b} / l_{B a}$ \\
\hline 3.125 & $5 \times 10^{-7}$ & 109 & 1.0 & 30.5 & 30.5 \\
\hline 3.125 & $5 \times 10^{-7}$ & 109 & 5.0 & 30.5 & 68.1 \\
\hline 3.125 & $5 \times 10^{-7}$ & 109 & 10.0 & 30.5 & 96.4 \\
\hline
\end{tabular}

smallest scales of turbulence, whereas the Batchelor $\left(l_{B a}\right)$ length scale [66] describes the smallest scales of fluctuations in concentration before molecular diffusion dominates:

$l_{K o}=\left(\frac{\nu_{c}^{3}}{\epsilon}\right)^{1 / 4}, \quad l_{B a}=\frac{l_{K o}}{\sqrt{S c}}$,

where $v_{c}=\mu_{c} / \rho_{c}$ is the kinematic viscosity in $\Omega_{c}$, and $\epsilon \approx g U_{T}$ approximates the rate of energy dissipation per unit mass,computed as the work performed by a gravity-driven bubble rising in a quiescent liquid $[29,1,67]$. Table 2 shows $d_{b} / l_{K o}$ and $d_{b} / l_{B a}$ for a typical case $(E o, M o, R e)$ computed in this research. It can be observed that Schmidt number $(S c)$ plays an important role to define the grid size. According to $l_{B a}$ it is necessary a grid size $h \sim d_{b} / 30$ for $S c=1$, and $h \sim d_{b} / 96$ for $S c=10$, in order to capture physical concentration gradients. Therefore, a selection $h=d_{b} / 35$ obtained by the previous grid refinement study, is consistent with the expected $l_{B a}$ and $l_{K o}$, for the range of dimensionless parameters used in this work.

\subsubsection{Effect of convective schemes}

An assessment of convective schemes (see Appendix A) is important to avoid numerical oscillations around discontinuities and to minimize the so-called numerical diffusion. Numerical experiments were performed to research the influence of the convective scheme on the discretization of momentum equation (Eq. (18)), and concentration equation (Eq. (17)). Consistently with Section 4.3.1, the unstructured mesh $M_{1}$ (Table 1 ) is used in this section.

Fig. 8 depicts the time evolution of Reynolds number $(R e)$, normalized surface of the bubble $\left(A^{*}(t)=A(t) / A(0)\right)$ with $A(t)=\int_{\Omega}\|\nabla \phi\| d V$, Sherwood number (Sh), and the concentration (C) profile along the symmetry axis of $\Omega$ (y-axis) at $t^{*}=3.8$, for
$E o=3.125, M o=5 \times 10^{-6}, S c=1, \eta_{\rho}=100, \eta_{\mu}=100$. Fig. 8 a illustrates numerical results using a Superbee convective scheme for the mass transfer equation (Eq. (12)), whereas Superbee, Centraldifference (CD), Upwind and Smart convective schemes are used for momentum equation (Eq. (1)). It is observed that $\operatorname{Re}(t) \mathrm{com}-$ puted by an Upwind scheme is lower than results obtained with other flux limiters. In addition, concentration profiles calculated by Upwind, Smart and CD limiters are delayed in comparison with that computed using a Superbee flux-limiter scheme. Fig. 8b illustrates numerical results using a Superbee convective scheme for momentum equation, whereas Superbee, CD, Upwind and Smart convective schemes are used for mass transfer equation. Here, it can be observed that computation of Sherwood number is sensitive to the employed flux-limiter. Moreover, Fig. 9, shows that concentration contours computed by using an Upwind scheme lead to numerical diffusion, whereas results with Superbee flux-limiter minimize the aforementioned numerical effect. As a consequence, in what follows Superbee flux-limiter scheme is selected to discretize convective terms. The reader is referred to the Appendix A for further technical details on the implementation of unstructured flux-limiters employed in this work.

\subsubsection{Prediction of $\operatorname{Re}=\operatorname{Re}(E o, M o)$ and $C_{D}=C_{D}(E o, R e)$}

A set of numerical experiments is performed to investigate the interaction between hydrodynamics and external mass transfer from a single bubble. Dimensionless parameters are $0.5 \leqslant$ Eo $\leqslant 3.125$ and $10^{-8} \leqslant M o \leqslant 5 \times 10^{-2}$, which correspond to spherical and ellipsoidal bubbles according to the Grace diagram [5]. Additional parameters are defined as $\eta_{\rho}=100, \eta_{\mu}=100$, $D a=0$ and $S c=1$. The computational set-up, initial condition and boundary conditions are defined as in Section 4.3.1. Further- 
(a)

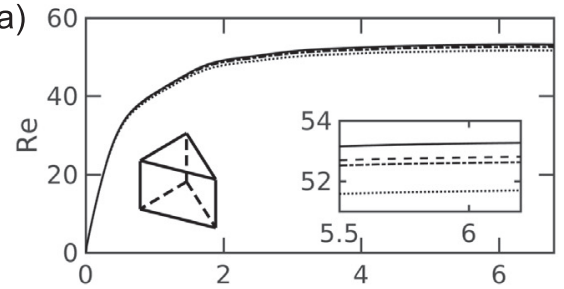

$t^{*}$
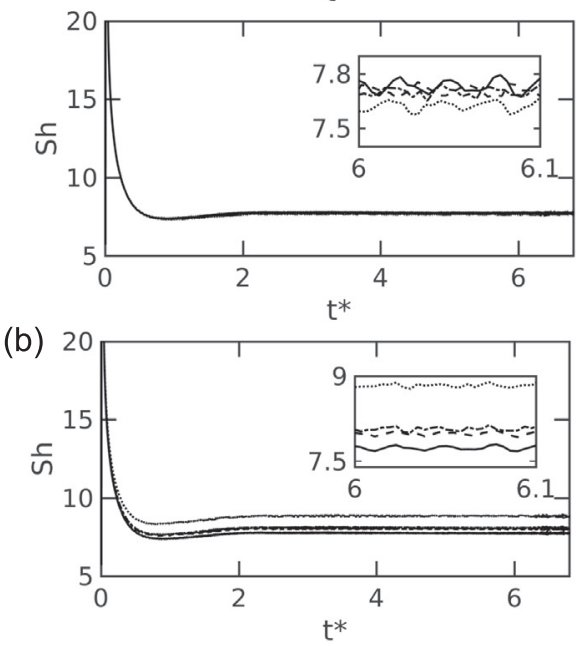

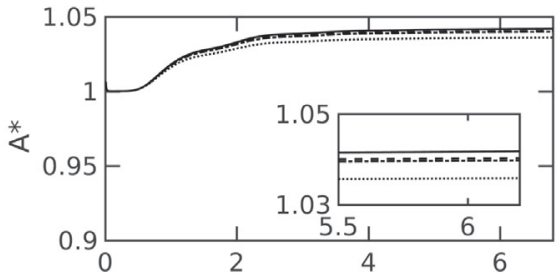

t*
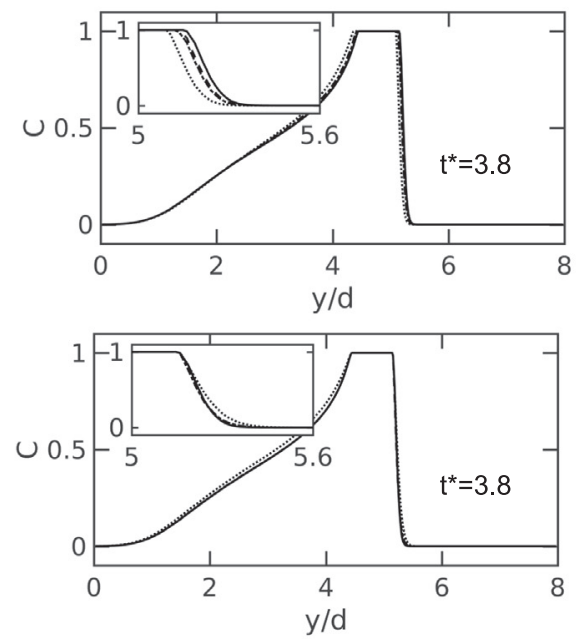

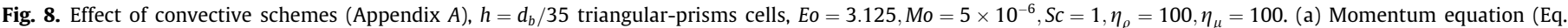

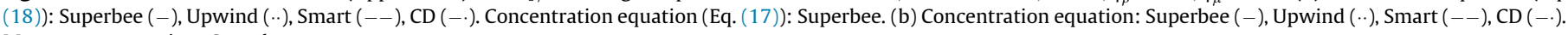
Momentum equation: Superbee.


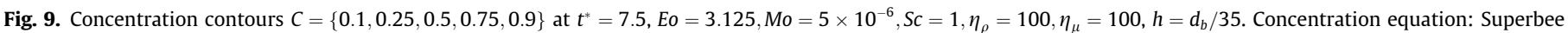
$(-)$, Upwind (..), Smart (--), CD (-.). Momentum equation: Superbee.

more, the spatial domain $\Omega$, and the meshes used in this section are summarized in Table 1.

Fig. 10 depicts the time evolution of Reynolds number, normalized bubble surface, and Sherwood number. Fig. 10b illustrates results calculated on the mesh $M_{4}$ (Table 1), whereas results depicted in Fig. 10a-c-d were calculated on the mesh $M_{1}$ (Table 1). Since physical properties ratios are fixed to $\eta_{\rho}=100$ and $\eta_{\mu}=100$, then $\operatorname{Re}=\operatorname{Re}(E o, M o)$. As can be observed from Fig. 10, Re achieves its terminal value after a short period of time. If Eo is kept constant, $R e$ increases as Mo decreases; whereas if Mo is kept constant, Re increases as Eo increases. The normalized bubble surface is equal to unity at the initial state, which correspond to a spherical bubble. As simulations advance, the bubble surface increase as a consequence of the bubble deformation.

Since the mass transfer rate $(S h=S h(R e, S c))$ depends of the bubble hydrodynamics $(R e)$; as a first step it will be validated by comparing the drag-force coefficient $\left(C_{D}=C_{D}(R e, E o)\right)$ and $\operatorname{Re}=\operatorname{Re}(W e, M o)$, against correlations from the literature. For a rising bubble $C_{D}$ can be obtained from a steady-state force balance, $F_{\text {buoyant }}+F_{\text {drag }}=0$, in the direction $\hat{\mathbf{e}}_{y}$ : $\left(\frac{1}{6} \pi d_{b}^{3}\right)\left(\rho_{c}-\rho_{d}\right) \hat{\mathbf{e}}_{y} \cdot \mathbf{g}-C_{D} \frac{\pi d_{b}^{2}}{4} \frac{\rho_{c}}{2}\left(\left(\overline{\mathbf{v}}_{d}-\overline{\mathbf{v}}_{c}\right) \cdot \hat{\mathbf{e}}_{y}\right)^{2}=0$,

where $\overline{\mathbf{v}}_{d}=T^{-1} \int_{t_{0}}^{t_{0}+T} \mathbf{v}_{d}(t) d t$ is the time-averaged velocity during the period $T$ at steady-state, with a similar definition for $\overline{\mathbf{v}}_{C}$. The previous force balance leads to a practical formula for $C_{D}$, namely:

$C_{D}=\frac{4 d_{b}\left(\rho_{c}-\rho_{d}\right) \hat{\mathbf{e}}_{y} \cdot \mathbf{g}}{3 \rho_{c}\left(\left(\overline{\mathbf{v}}_{d}-\overline{\mathbf{v}}_{c}\right) \cdot \hat{\mathbf{e}}_{y}\right)^{2}}$.

Dijkhuizen et al. [68] proposed a drag closure for pure liquids, $C_{D}=C_{D}(R e, E o)$, valid for spherical and deformed bubbles, which has the form:

$$
\begin{aligned}
& C_{D}=\sqrt{C_{D}(R e)^{2}+C_{D}(E O)^{2}}, \\
& C_{D}(R e)=\frac{16}{R e}\left(1+\frac{2}{1+\frac{16}{R e}+\frac{3.315}{\sqrt{R e}}}\right), \\
& C_{D}(E O)=\frac{4 E o}{9.5+E 0} .
\end{aligned}
$$

Please cite this article as: N. Balcázar-Arciniega, O. Antepara, J. Rigola et al., A level-set model for mass transfer in bubbly flows, International Journal of Heat and Mass Transfer, https://doi.org/10.1016/j.ijheatmasstransfer.2019.04.008 
(a)
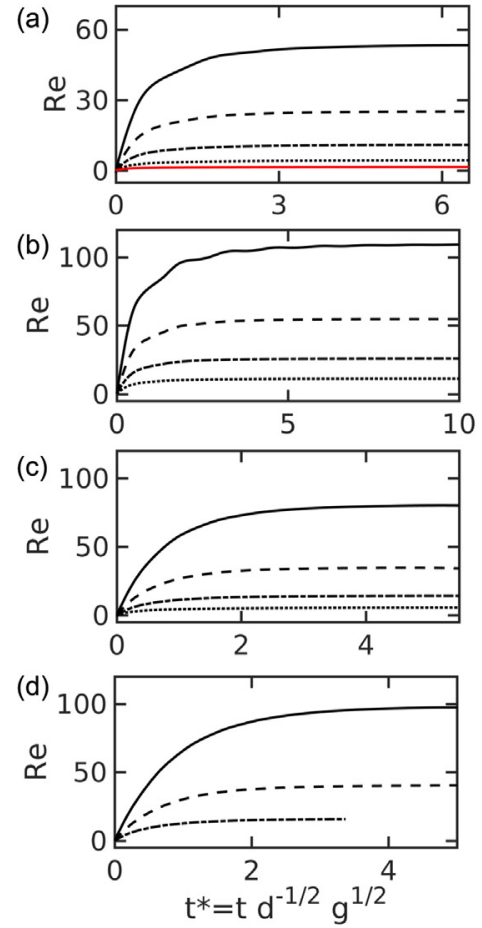
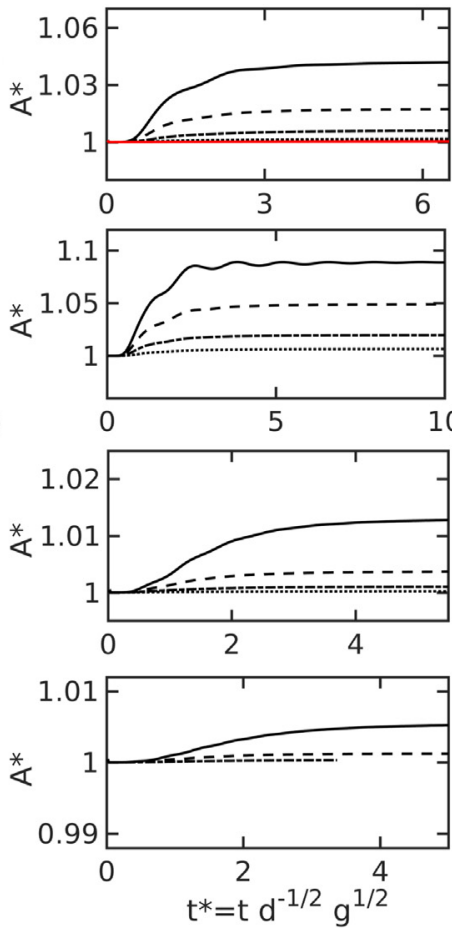


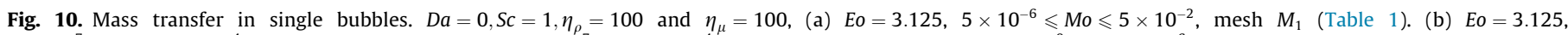
$5 \times 10^{-7} \leqslant M o \leqslant 5 \times 10^{-4}$, mesh $M_{4}$ (Table 1). (c) Eo $=1.0,10^{-7} \leqslant M o \leqslant 10^{-4}$, mesh $M_{1}$ (Table 1). (d) Eo $=0.5,10^{-8} \leqslant M_{0} \leqslant 10^{-6}$, mesh $M_{1}$ (Table 1 ).

Furthermore, Rastello et al. [69] proposed a correlation $\operatorname{Re}=\operatorname{Re}(W e, M o)$, in terms of Weber number $W e=\rho_{c} U_{T}^{2} d_{b} / \sigma$ and Morton number $M o=\mu_{c}^{4} g /\left(\sigma^{3} \rho_{c}\right)$, as follows:

$\operatorname{Re}=2.05 \mathrm{We}^{2 / 3} \mathrm{Mo}^{-1 / 5}$.

Fig. 11a shows that numerical results for $C_{D}$ computed by the presented method, match fairly well with the correlation (Eq. (39)) proposed by Dijkhuizen et al. [68], for $E o=\{0.5,1,3.125\}$. It is observed an increment in $C_{D}$ as the Eo increases; whereas the range of the drag force coefficient is maintained in $0.1<C_{D}<20$ for Reynolds numbers $1<R e<150$. Fig. $11 \mathrm{~b}$ shows that all numerical results expressed in terms of Re, We and Mo, closely fit with the correlation (Eq. (40)) proposed by Rastello et al. [69]. Furthermore, Fig. 11c illustrates some examples of vorticity contours $\left(\omega_{z}=\hat{\mathbf{e}}_{z} \cdot(\nabla \times \mathbf{v})\right)$ on the plane $x-z$, as well as the final bubble shapes, for $E o=3.125$ and $10^{-7} \leqslant M o \leqslant 10^{-4}$. These results validate the hydrodynamics on three-dimensional unstructured meshes.

\subsubsection{Prediction of $S h=S h(R e, S c)$}

As a further step, the computed Sherwood numbers summarized in Fig. 10, are compared with available correlations from the literature. Multiple models of the Sherwood number, $S h=S h(R e, S c)$, in single bubbles rising in a quiescent liquid have been proposed, for spherical and deformed bubble shapes. For instance, Boussinesq [70] proposed a theoretical model for mass transfer from a single spherical bubble in the limit of potential flow, considering that the transfer occurs across a very thin concentration layer at the bubble interface:

$S h=\frac{2}{\sqrt{\pi}} P e^{1 / 2}$.

Winnikow [71] derived an expression for spherical bubbles with fully developed internal circulation, that includes the effect of Reynolds number:
$S h=\frac{2}{\sqrt{\pi}}\left(1-\frac{2.89}{\sqrt{R e}}\right)^{1 / 2} P e^{1 / 2}$,

which is valid for $R e>70$ [5]. Takemura and Yabe [72] proposed a correlation for spherical gas bubbles, $R e<100$ and $P e>1$ :

$S h=\frac{2}{\sqrt{\pi}}\left(1-\frac{2}{3} \frac{1}{\left(1+0.09 R e^{2 / 3}\right)^{3 / 4}}\right)^{1 / 2}(2.5+\sqrt{P e})$.

Lochiel and Calderbank [73] reported an expression for oblate spheroidal bubbles, considering a potential flow around the bubble:

$S h=\frac{2}{\sqrt{\pi}} \sqrt{P e}\left(\frac{2}{3}(1+k)\right)^{1 / 2} \frac{2 \chi^{1 / 3}\left(\chi^{2}-1\right)^{1 / 2}}{\chi\left(\chi^{2}-1\right)^{1 / 2}+\ln \left(\chi+\sqrt{\chi^{2}-1}\right)}$,

where $\chi$ is the bubble aspect ratio, $k$ is the velocity factor, and $e$ is the eccentricity defined as follows:

$\chi=\frac{\text { major axis }}{\text { minor axis }}, \quad k=-\left(\frac{e \chi^{2}-\chi \sin ^{-1} e}{e-\chi \sin ^{-1} e}\right), \quad e=\sqrt{1-\frac{1}{\chi^{2}}}$.

Numerical results for the computed Sherwood numbers $(2<S h<15)$ versus Reynolds numbers $(1<R e<120)$, as well as results predicted by correlations from the literature, are depicted in Fig. 12a-b, with $S c=1$ and $D a=0$. Numerical results computed on the triangular-prisms mesh $M_{1}$ (Table 1 ) for $E o=\{0.5,1,3.125\}$, and hexahedral mesh $M_{4}$ (Table 1 ) for $E o=\{3.125\}$, demonstrate that the level-set model leads to similar accuracy independently of the cell geometry. As shown in Fig. $10\left(A^{*}(t)\right)$ and Fig. 12c (e.g., $\left.(E o, M o)=\left\{\left(0.5,10^{-6}\right),\left(3.125,10^{-3}\right)\right\}\right)$ the bubble shapes tend to be spherical for the lowest Reynolds numbers, and deformed (ellipsoidal) for the highest Reynolds numbers. Consistently, our numerical results are closer to predictions of Takemura and Yabe 

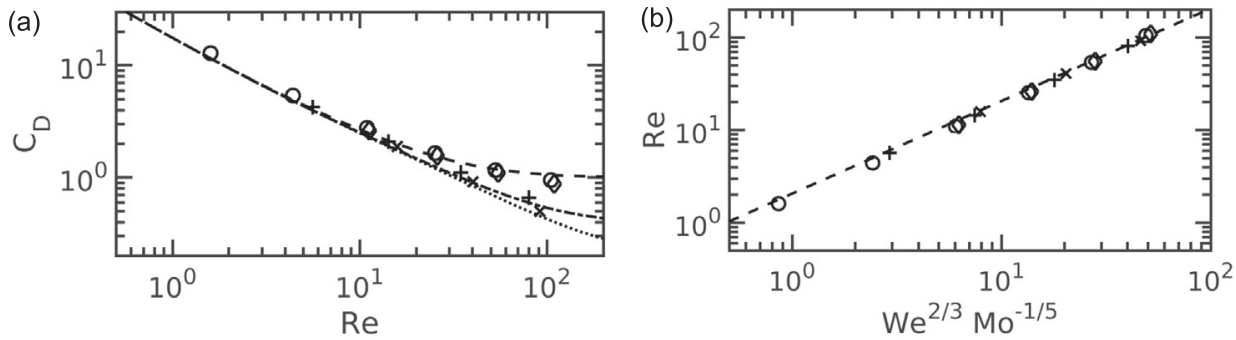

(c)
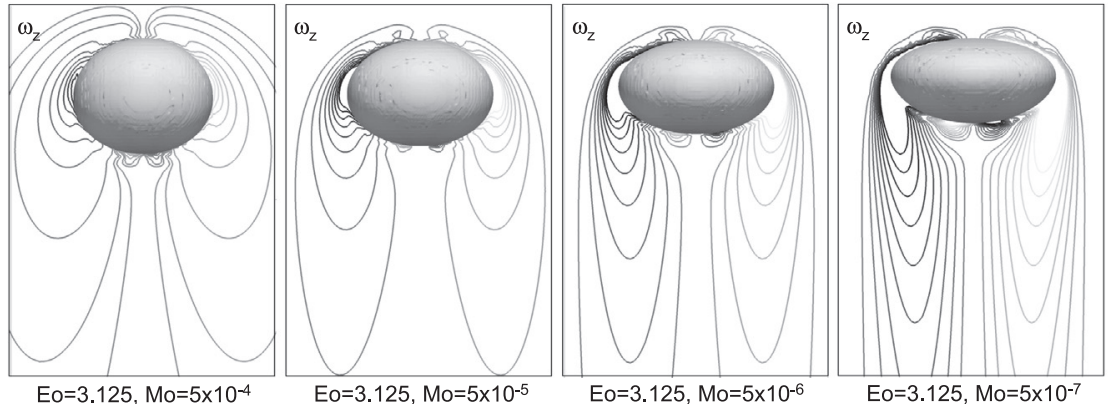

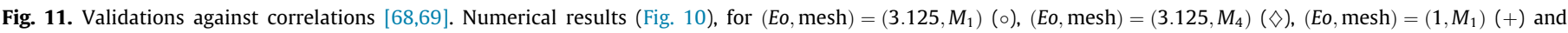


(c) Contours $\omega_{z}=\hat{\mathbf{e}}_{z} \cdot \nabla \times \mathbf{v}$.
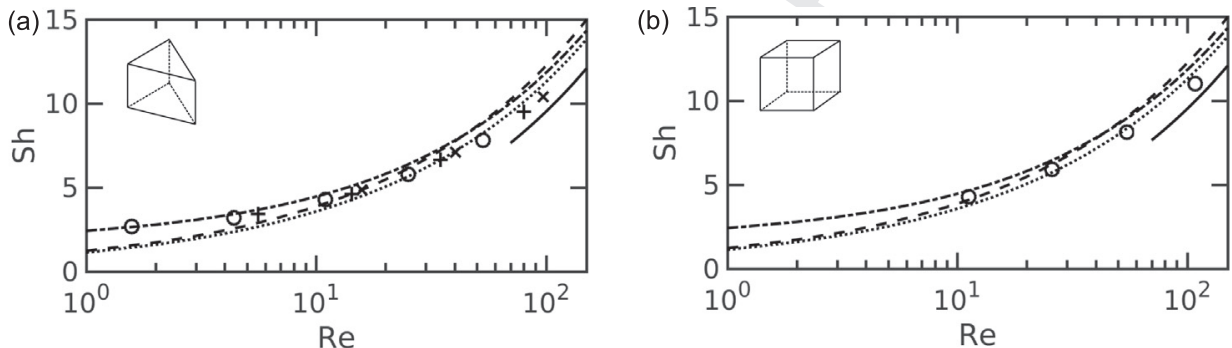

(c)
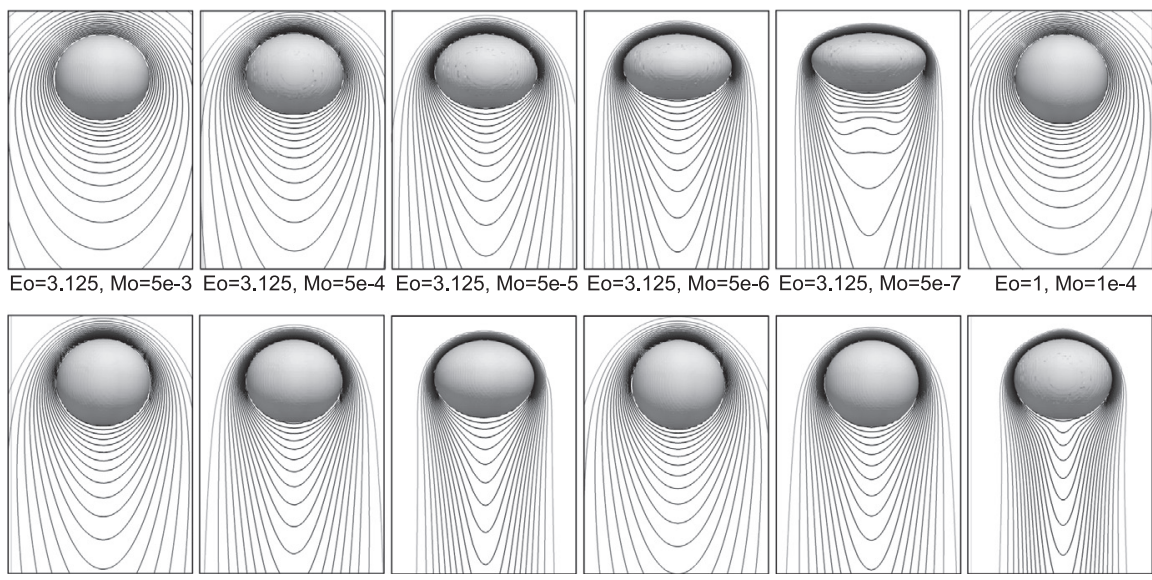

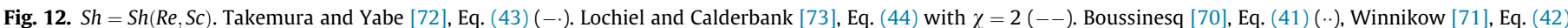

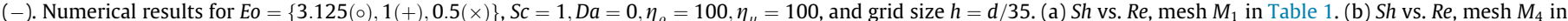
Table 1. (c) Concentration contours, mesh $M_{1}$ in Table 1.

[72] correlation (Eq. (43)) for spherical bubbles at low Re; whereas numerical predictions are closer to Lochiel and Calderbank [73] correlation for deformed bubbles at high $R e$. As a further validation, Fig. 13 shows the effect of Schmidt number on the Sherwood number for $E o=3.125, M o=5 \times 10^{-4}$ and $R e=11$. These simulations were performed on the mesh $M_{1}$ (Table 1) with triangular-prism cells. Fig. 13a depicts the time evolution of $\mathrm{Sh}$ for
$S c=\{1,5,10,20,50\}$, whereas Fig. $13 \mathrm{~b}$ shows Sh versus Sc. It can be observed that numerical predictions are closer to the correlation of Takemura and Yabe [72] for the highest Sc. Fig. 13c illustrates the effect of $S c$ on the concentration field. As it is expected, the thickness of the concentration boundary layer is reduced as the Schmidt number increases, whereas the concentration tends to be sharpest concentrated in the wake of the bubble as $S c$ increases. 

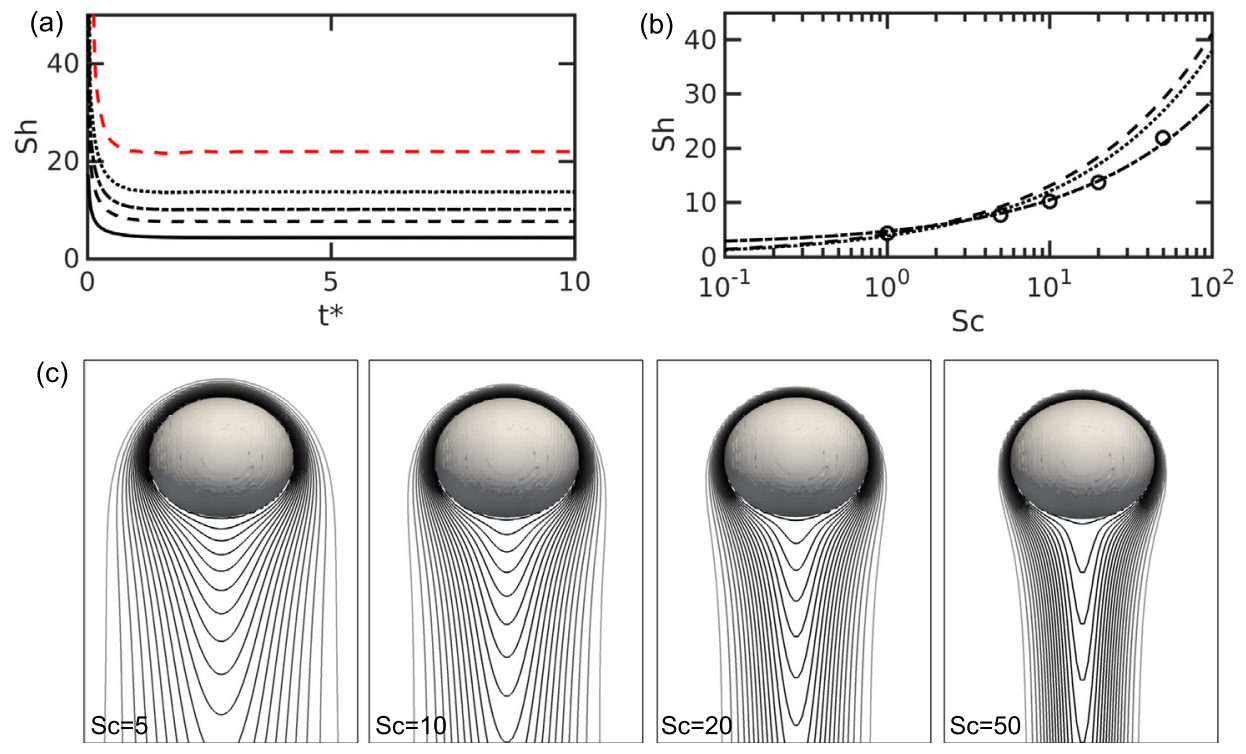



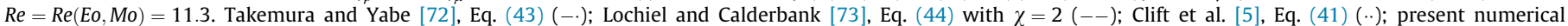
results $(\circ)$. (c) Concentration contours. (For interpretation of the references to colour in this figure legend, the reader is referred to the web version of this article.)

Therefore, results of these numerical experiments validate the proposed level-set model for mass transfer coupled to the hydrodynamics.

\subsection{Mass transfer from bubble swarms}

This section aims to explore the interaction of fluid mechanics and mass transfer from bubble swarms rising on a stable bubble column, where there is no gradient of bubble fraction and no large-scale liquid motions induced by buoyancy. A sample of a whole bubble column is represented by a cubic domain with fully periodic boundaries, which means that once bubbles leave from one boundary, new bubbles come in through the opposite boundary. One of the simulations reported by Esmaeli and Tryggvason [52] is reproduced here for the sake of validation. The problem consists on the buoyancy-driven motion of a three-dimensional bubble, with $E o=2, M o=10^{-5}, \eta_{\rho}=10, \eta_{\mu}=10$, and bubble fraction $\alpha=0.1256 . \Omega$ is a cube with side-length $L_{\Omega}=1.61 d_{b}$, where $d_{b}$ is the initial bubble diameter.

Fig. 14a depicts the time evolution of the Reynolds number computed by the present model on a uniform hexahedral mesh of $\left\{50^{3}, 100^{3}, 150^{3}\right\}$ grid cells. The difference in the terminal Reynolds number computed by meshes $100^{3}$ and $150^{3}$ is $0.9 \%$, whereas it is $3.4 \%$ between meshes $50^{3}$ and $100^{3}$, which demonstrate grid convergence. For the sake of comparison, a sample of data reported by $[52,51]$ were extracted and plotted in Fig. 14. Simulations of Esmaeli and Tryggvason [52] were carried out by using the front-tracking method, whereas Coyajee and Boersma [51] used a coupled volume-of-fluid/level-set method. It is observed that time evolution of Reynolds number computed by the present method, is in close agreement with numerical results reported by [52,51]. Fig. 14b depicts a bubble at the steady state, and the streamlines in a reference frame moving with the bubble, computed on a $150^{3}$ mesh. Present results are consistent with those reported by Esmaeli and Tryggvason [52], which validates the present model on fully periodic domains.

\subsubsection{Prediction of $\operatorname{Re}=\operatorname{Re}(E o, M o, \alpha)$ and $C_{D, \text { swarm }}=C_{D}(\alpha, E o)$}

Bubbles are initially distributed in $\Omega$ following a random pattern, whereas both fluid phases are initially quiescent. Since fluids are incompressible and numerical coalescence is avoided, the bubble volume fraction $(\alpha)$ and number of bubbles $\left(n_{d}\right)$ are constant throughout the simulation. Dimensionless parameters are $E o=3.125, M o=\left\{5 \times 10^{-7}, 5 \times 10^{-8}\right\}, \quad \eta_{\rho}=100, \eta_{\mu}=100 \quad$ and $3 \%<\alpha<20 \%$ as summarized in Table 3. These simulations are performed on a fully periodic cube of $4 d_{b}$ side-length, discretized

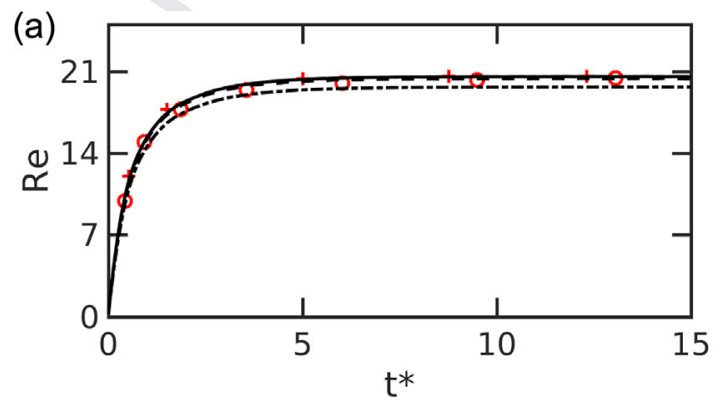

(b)

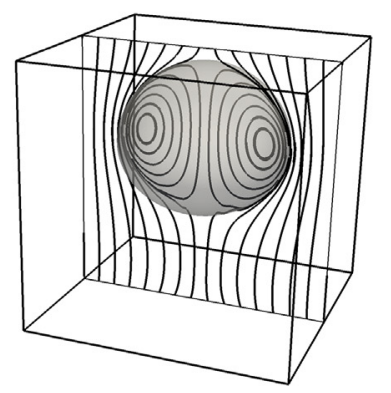

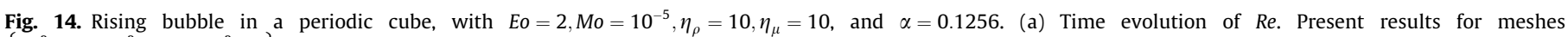
$\left\{50^{3}(-\cdot), 100^{3}(--), 150^{3}(-)\right\}$, Esmaeli and Tryggvason [52] (o), Coyajee and Boersma [51] (+). (b) Steady-state bubble shape and streamlines. 
Table 3

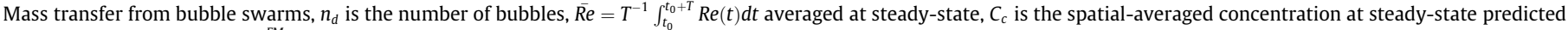
by the level-set model, and $C_{c}^{F M}$ is the concentration predicted by the film theory [45] (Eq. (54)).

\begin{tabular}{|c|c|c|c|c|c|c|c|c|c|c|}
\hline Case & Eo & Mo & Sc & $\alpha$ & $n_{d}$ & $D a$ & $\overline{R e}$ & $C_{c}$ & $C_{c}^{F M}$ & Sh \\
\hline 1 & 3.125 & $5 \times 10^{-8}$ & 1 & $3.27 \%$ & 4 & 25.2 & 163.2 & 0.1052 & 0.1038 & 13.9 \\
\hline 2 & 3.125 & $5 \times 10^{-8}$ & 1 & $8.18 \%$ & 10 & 25.2 & 138.5 & 0.2572 & 0.2574 & 15.1 \\
\hline 3 & 3.125 & $5 \times 10^{-8}$ & 1 & $9.82 \%$ & 12 & 25.2 & 130.2 & 0.2991 & 0.3002 & 15.6 \\
\hline 4 & 3.125 & $5 \times 10^{-8}$ & 1 & $13.09 \%$ & 16 & 25.2 & 118.6 & 0.3864 & 0.3898 & 16.6 \\
\hline 5 & 3.125 & $5 \times 10^{-8}$ & 1 & $16.36 \%$ & 20 & 25.2 & 107.3 & 0.4636 & 0.4692 & 16.8 \\
\hline 6 & 3.125 & $5 \times 10^{-8}$ & 1 & $19.63 \%$ & 24 & 25.2 & 99.6 & 0.5190 & 0.5275 & 17.9 \\
\hline 7 & 3.125 & $5 \times 10^{-7}$ & 1 & $9.82 \%$ & 12 & 14.2 & 75.6 & 0.3818 & 0.3849 & 12.7 \\
\hline
\end{tabular}

by a $150^{3}$ uniform hexahedral mesh, which is equivalent to the grid size $h=d_{b} / 37.5$.

Fig. 15 depicts the time evolution of Reynolds number (Eq. (26)), normalized surface for each bubble $A_{i}^{*}(t)=A_{i}(t) /\left(\pi d^{2}\right)$ with $A_{i}(t)=\int_{\Omega}\left\|\nabla \phi_{i}\right\| d V$, normalized total surface of bubbles $A^{*}(t)=\sum_{i}^{n_{d}} A_{i}(t) /\left(\pi d_{b}^{2}\right)$, bubble trajectories projected on the plane $z-y$, spatial-averaged concentration of the chemical species $\left(C_{c}(t)\right)$, and $S h(t)$ computed by Eqs. (28) and (52); whereas simulations correspond to cases $3-5$ from Table 3. Fig. 16a shows $\operatorname{Re}(t)$
(Eq. (26)) of the bubble swarm for all cases of Table 3. Regarding the hydrodynamics, it is observed from Fig. 15 that $\operatorname{Re}_{i}(t)$ (Eq. 25) presents fluctuations originated by the bubble-bubble interactions and bubble shape oscillations. Nevertheless, the time-averaged Reynolds number $\left(T^{-1} \int_{t_{0}}^{t_{0}+T} \operatorname{Re}(t) d t\right)$ tends to the steady-state as the simulation advances (discontinuous gray lines in Fig. 15), whereas it tends to decreases as the bubble fraction increases.

The drag-force coefficient defined in Eq. (38) for a single bubble, should be modified for bubble swarms in order to account the effect of local bubble fraction $(\alpha)$. Thus, following the work of Rog-
980

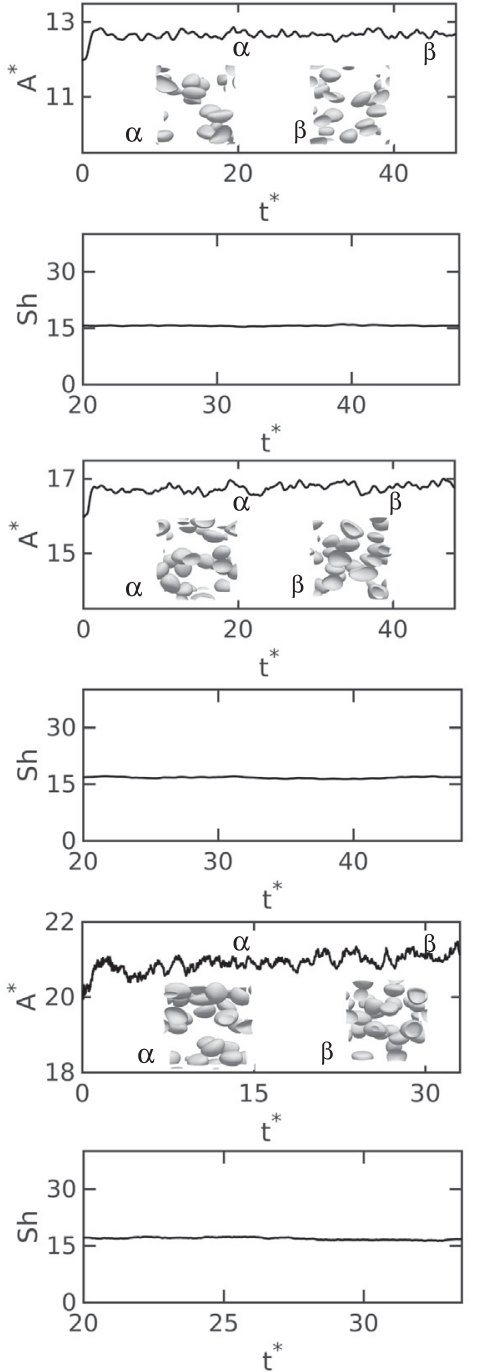

Fig. 15. Mass transfer from bubble swarms. (a) Case 3 from Table 3. (b) Case 4 from Table 3. (c) Case 5 from Table 3. 

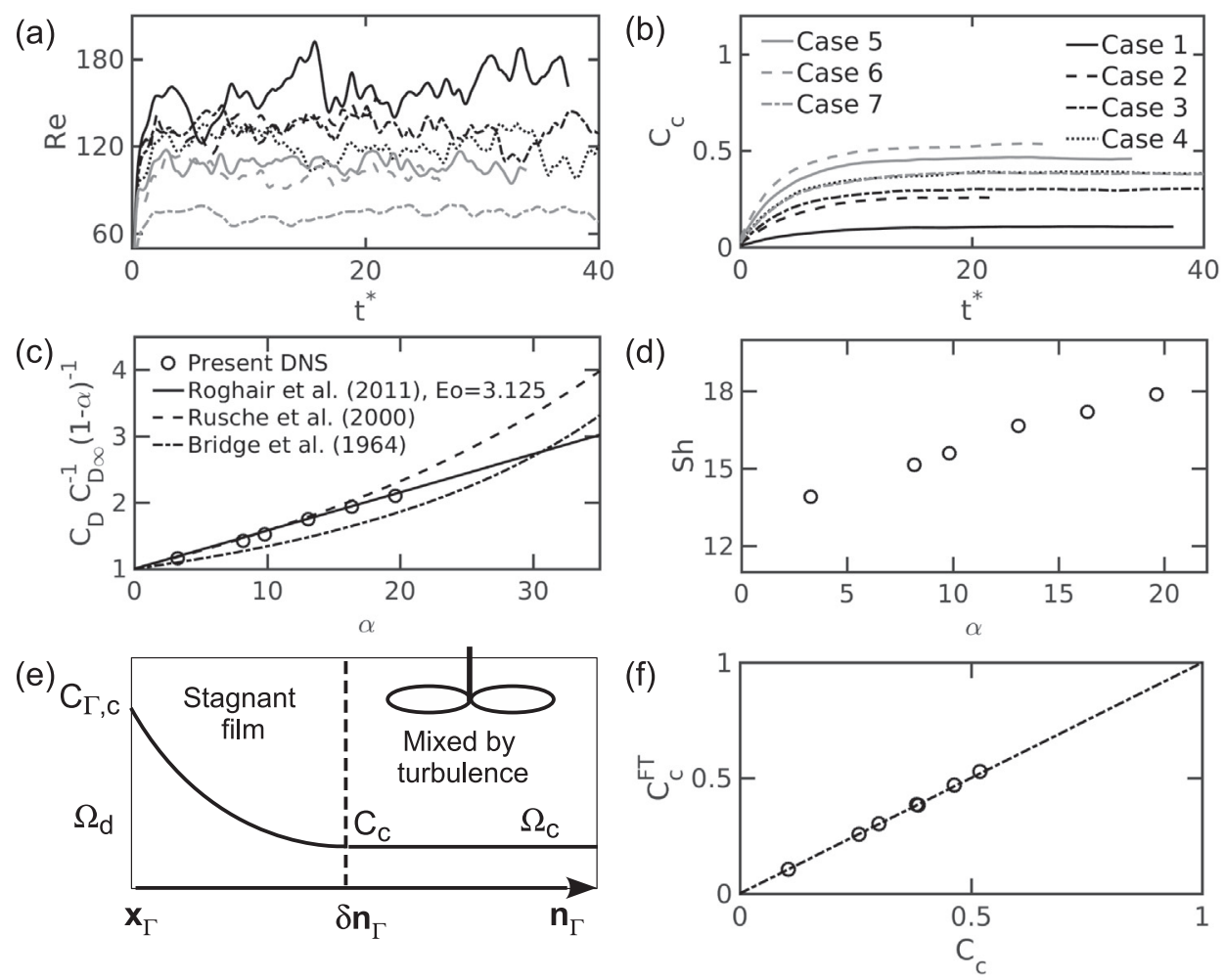

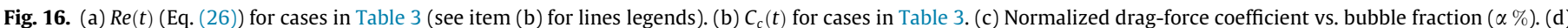

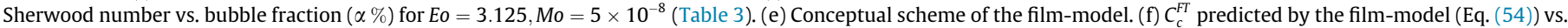
$C_{c}$ predicted by present DNS (Table 3 ).

hair et al. [74] and Simonnet et al. [76] the drag-force coefficient for a bubble in a swarm reads:

$C_{D, \text { swarm }}=\frac{4 d_{b}\left(\rho_{c}-\rho_{d}\right) \hat{\mathbf{e}}_{y} \cdot \mathbf{g}}{3 \rho_{c}\left(\left(\overline{\mathbf{v}}_{d}-\overline{\mathbf{v}}_{c}\right) \cdot \hat{\mathbf{e}}_{y}\right)^{2}}(1-\alpha)$.

Furthermore, some correlations for the drag-force coefficient in a swarm of bubbles ( $\left.C_{D, \text { swarm }}\right)$ have been reported in literature [7477]. Following the approach of Roghair et al. [74] and Rusche and Issa [77] a correction function $f(\alpha)$ can be used to account the effect of bubble fraction on $C_{D, s w a r m}$, in comparison to the single rising bubble drag-force coefficient $C_{D, \infty}$ (see Eq. (38)), as follows:

$\frac{C_{D, \text { swarm }}}{C_{D, \infty}(1-\alpha)}=f(\alpha)$.

In this context, a correction function was proposed by Rusche and Issa [77] for bubbly flows, based on experimental data:

$f(\alpha)=\exp (3.64 \alpha)+\alpha^{0.864}$

Bridge et al. [75] proposed an exponent $n=1.29$ for the correlation:

$f(\alpha)=(1-\alpha)^{2 n}$.

Roghair et al. [74] developed a drag closure for mono-disperse bubble swarms, in the range of $1 \leqslant E 0 \leqslant 5$ and $10^{-11}<M o<10^{-9}$, based on DNS data:

$f(\alpha)=1+g(E o) \alpha=1+\frac{18}{E o} \alpha$.

Fig. 16c shows that the normalized drag-force coefficients, for cases $(E o, M o)=\left(3.125,5 \times 10^{-8}\right)$ in Table 3, adjust to a linear relation with the bubble fraction; whereas present simulations predict the same order of magnitude for $f(\alpha)$ (Eq. (47)) than those predicted by correlations from literature $[77,75]$. Furthermore, it is observed from Fig. 16c that our results compares fairly well with the correlation proposed by Roghair et al. [74] for Eo $=3.125$ (Eq. (50)); which validates the proposed level-set model for the hydrodynamics of bubble swarms.

\subsubsection{Prediction of $\operatorname{Sh}=\operatorname{Sh}(\operatorname{Re}, S c, D a, \alpha)$}

In this section, numerical results of mass transfer from bubble swarms coupled to their hydrodynamics are discussed, for cases summarized in Table 3. Since mass transfer simulations are carried out in a periodic domain, the concentration will be saturated $\left(C_{c}=C_{\Gamma, c}\right)$ if there is no consumption of the chemical species transferred to $\Omega_{c}$. As introduced by Roghair et al. [45], the aforementioned problem is avoided by activating the chemical reaction term $(D a>0)$ in the mass transfer equation (Eq. (12)). Thus, for a batch reactor at steady state, the mass balance between the mass transfer from bubbles and a first-order chemical reaction in $\Omega_{c}$ is written as follows:

$V_{c} \frac{d C_{c}}{d t}=k_{c} \sum_{i=1}^{n_{d}} A_{i}\left(C_{\Gamma, c}-C_{c}\right)-k_{1} C_{c} V_{c}=0$,

where $A_{i}=\int_{\Omega}\left\|\nabla \phi_{i}\right\| d V$ is the interfacial area for each bubble, $C_{\Gamma, c}$ is the concentration at the interface from the liquid-side $\Omega_{c}$, and $C_{c}=V_{c}^{-1} \int_{\Omega_{c}} C d V$ is the spatial-averaged concentration of the chemical species in $\Omega_{c}$. From the previous balance, the mass transfer coefficient can be computed for the steady-state as follows:

$k_{c}=\frac{k_{1} V_{c} C_{c}}{\left(C_{\Gamma, c}-C_{c}\right) \sum_{i=1}^{n_{d}} \int_{\Omega}\left\|\nabla \phi_{i}\right\| d V}$.

Therefore, for reactive mass transfer, $k_{c}$ is a function of the chemical reaction constant $\left(k_{1}\right)$, the averaged concentration $\left(C_{c}\right)$, and interfacial surface. In order to report numerical results, the Sherwood number (see Eq. (28)) will be employed in place of $k_{c}$. 
Fig. 15 (see $C_{c}(t)$ ) and Fig. 16b shows that after initial transient effects, the spatial-averaged concentration of the chemical species $\left(C_{c}\right)$ achieves the steady-state, indicating a balance between the mass transfer rate and the consumption of the chemical species (Eq. (51)). At this point, it is possible to use Eq. (52) to compute the Sherwood number, as illustrated in Fig. 15. Further numerical results for the predicted Sherwood number $(S h)$ are summarized in Table 3 and Fig. 16d. It can be observed a linear relation between Sh and $\alpha$, whereas $S h$ increases with the bubble-volume fraction (3\%< $<<20 \%$ ). This trend is consistent with numerical findings reported by Roghair et al. [45] in the framework of a fronttracking methodology.

Figs. 17 and 18 show snapshots of the bubble distribution for cases summarized in Table 3, as well as concentration field and
1063

1064

1065

1066

1067

1068

1069 (a)
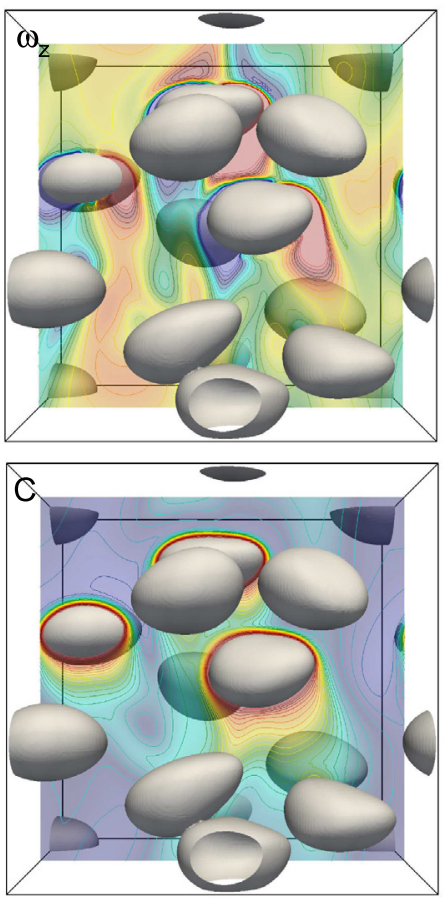

(b)
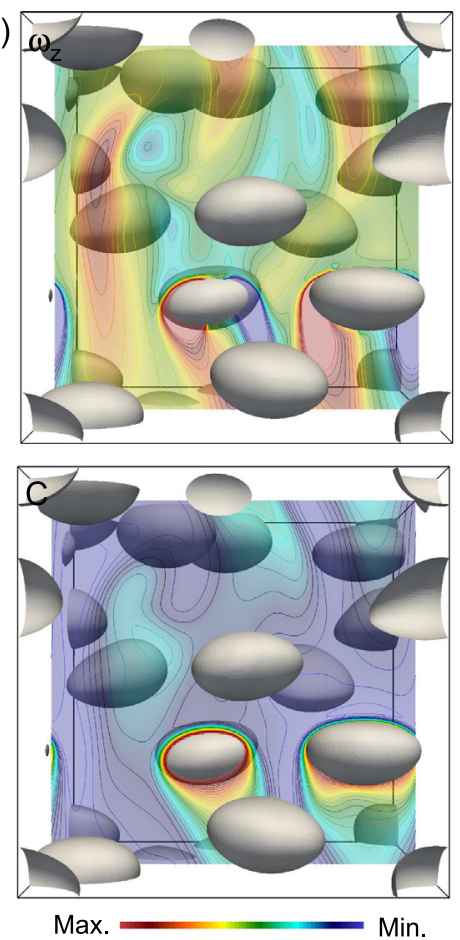

(c)


Fig. 17. Mass transfer from a swarm of 12 bubbles in a fully periodic box, $E o=3.125, M o=5 \times 10^{-7}, \eta_{\rho}=100, \eta_{\mu}=100, \alpha=0.1256, S c=1, D a=14.2$. Concentration contours $(C)$, and vorticity contours $\left(\omega_{z}=\hat{\mathbf{e}}_{z} \cdot \nabla \times \mathbf{v}\right)$. (a) $t^{*}=t \sqrt{g / d_{b}}=31.3$. (b) $t^{*}=34.5$. (c) $t^{*}=50.1$.

(a)
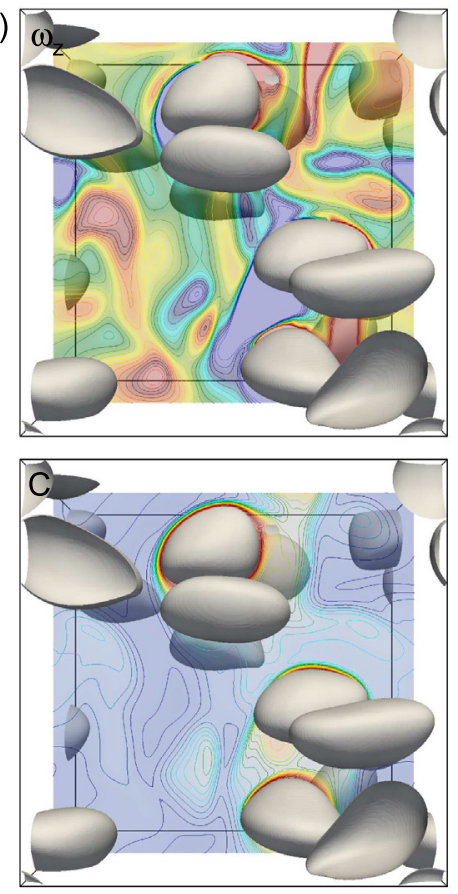

(b)
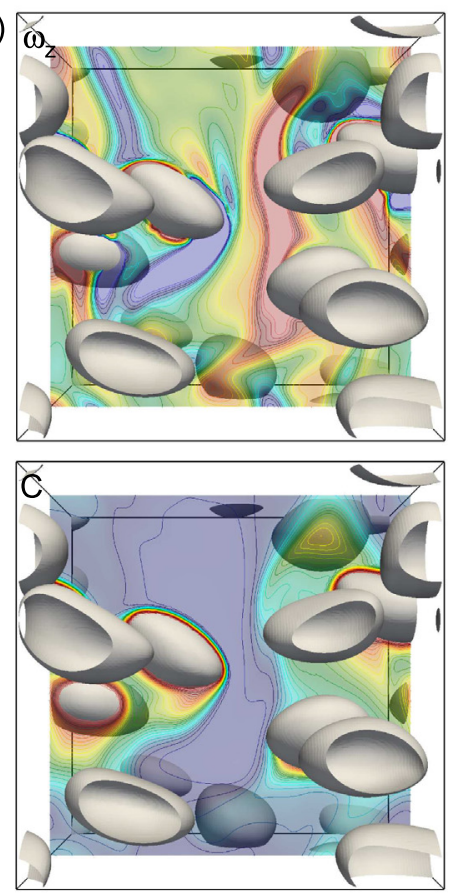

Max.
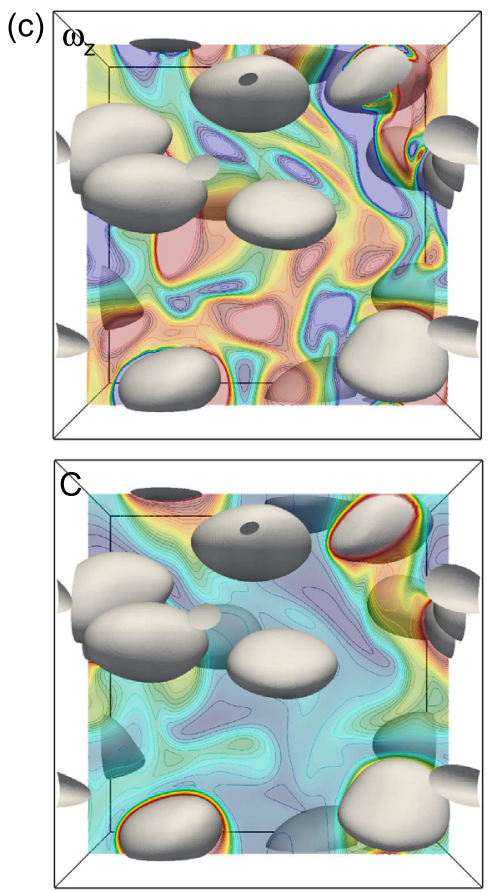

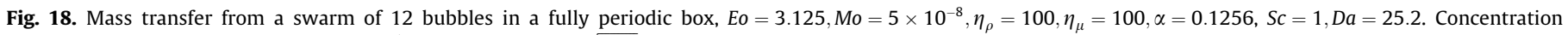
contours $(C)$, and vorticity contours $\left(\omega_{z}=\hat{\mathbf{e}}_{z} \cdot \nabla \times \mathbf{v}\right)$. (a) $t^{*}=t \sqrt{g / d_{b}}=18.8$. (b) $t^{*}=37.6$. (c) $t^{*}=57.4$. 
vorticity field generated by the interaction of the bubbles, at $(E o, M o, R e)=\left(3.125,5 \times 10^{-7}, 75.6\right)$ and $(E o, M o, R e)=\left(3.125,5 \times 10^{-7}, 120\right)$, respectively. As qualitatively illustrated in these figures, vorticity fields suggest that agitation tends to be stronger for higher Reynolds numbers, which is reflected also on the spatial distribution of concentration contours.

As a further validation test, the concentration predicted by the proposed level-set model will be compared with predictions of the so-called film theory. This consistency test was proposed by Roghair et al. [45] in the framework of a front-tracking method. In the film theory, it is assumed a stagnant film layer at the interface and a well-mixed bulk, as schematically illustrated in Fig. 16e. The stagnant layer has negligible capacitance and hence a local steady-state exists. In this context, the mass balance is written in a differential volume of the liquid boundary layer, where only diffusion and chemical reaction take place:

$\mathcal{D}_{c} \frac{d^{2} C(x)}{d x^{2}}=k_{1} C(x)$.

The boundary condition at the interface is $C(0)=C_{\Gamma, c}$, whereas at the limit of the liquid film and liquid bulk $(x=\delta)$, concentration is that of the liquid bulk $C(\delta)=C_{c}$. Furthermore, it is assumed batch operation for the integral mass balance (Eq. (51)). Following the work of Roghair et al. [45], the solution of that model can be expressed in terms of the Hatta number $\mathrm{Ha}$ ) and the Hinterland ratio $(A L)$ as follows [45]:

$C_{c}^{F T}=C_{\Gamma, c} \frac{1}{H a(A L-1) \sinh (H a)+\cosh (H a)}$

$H a=\left(\frac{k_{1} \mathcal{D}_{c}}{k_{c}^{2}}\right)^{1 / 2}, \quad A L=\frac{(1-\alpha) k_{c} V_{\Omega}}{\mathcal{D}_{c} \sum_{i=1}^{n_{d}} \int_{\Omega}\left\|\nabla \phi_{i}\right\| d V}$.

Fig. 16e shows that concentrations $\left(C_{c}\right)$ predicted by the present level-set model, match fairly well with concentrations predicted by the film theory $\left(C_{c}^{F T}\right)$.

\section{Conclusions}

A novel multiple-marker CLS approach has been introduced for mass transfer (or heat transfer) in single bubbles and bubble swarms. The method is based on a mass conservative level-set method for interface capturing, combined with a multiple marker approach to avoid the numerical coalescence of bubbles. The mathematical model has been solved using a finite-volume approach on a collocated unstructured mesh, including unstructured fluxlimiters schemes for convective terms in momentum, mass transfer and level-set advection equations. To the best of authors' knowledge, this is the first time that a multiple marker CLS methodology is presented for mass transfer in bubbly flows.

Numerical experiments demonstrate the ability of the method for simulating bubbly flows with mass transfer and first-order chemical reaction. Present results compare very well with analytical results and empirical correlations from the literature. Since the constraint imposed by the Batchelor length-scale (see Eq. (36)), present simulations are focused on low Schmidt numbers to achieve a practical grid-size for mass transfer and hydrodynamics phenomena, with Peclet number $(P e=S c R e \sim O(100))$.

Simulations of bubble swarms in a fully periodic domain show oscillations on the Reynolds numbers of each bubble, analogous to that observed in turbulence, which are induced by bubble-bubble interactions. Nevertheless, the time-averaged Reynolds number tends to the steady-state. These fluctuations are also observed on the bubble surfaces. Present numerical results show an increase of the Sherwood number with the bubble fraction, which is consistent with numerical findings reported by Roghair et al. [45].

Altogether, this research has introduced a predictive methodology based on first physical principles, for simulating the interaction of mass transfer and fluid mechanics, as well as to compute $\operatorname{Re}=\operatorname{Re}(E o, M o, \alpha), C_{D, \text { swarm }}=C_{D}(\operatorname{Re}, E o, \alpha)$ and $\operatorname{Sh}=\operatorname{Sh}(\operatorname{Re}, S c, D a, \alpha)$ in bubbly flows.

\section{Conflict of interest}

None.

\section{Acknowledgments}

This work has been financially supported by the Ministerio de Economía y Competitividad, Secretaría de Estado de Investigación, Desarrollo e Innovación (MINECO), Spain (ENE2015-70672-P), and by Termo Fluids S.L. The author Néstor Balcázar acknowledges the financial support of the Programa Torres Quevedo, MINECO (PTQ14-07186), Spain. Oscar Antepara acknowledges the support of MINECO (DI-14-06886) and Generalitat de Catalunya (2015DI-68), Spain. 3D simulations were performed using computing time awarded by PRACE 14th-Call (Ref. 2016153612), and RES (FI2018-1-0025), on the supercomputer MareNostrum IV based in Barcelona-Spain. Computer resources provided by RES (FI-20183-0037) on the supercomputer Altamira, supported by Santander Supercomputing group at the University of Cantabria (IFCA-CSIC), are acknowledged.

\section{Appendix A. Finite-volume schemes}

Spatial discretization of the governing equations is performed using the finite-volume method on a collocated unstructured mesh $[10,49]$. For the sake of completeness and for computer-code development, some points are remarked.

\section{A.1. Convective schemes}

The convective term of governing equations (or compressive term in Eq. (6)) is discretized at the current cell $\Omega_{P}$ as follows:

$\left(\nabla_{h} \cdot \beta \psi \mathbf{c}\right)_{P} \approx \frac{1}{V_{P}} \sum_{f} \beta_{f} \psi_{f} \mathbf{c}_{f} \cdot \mathbf{A}_{f}$

where $V_{P}$ is the volume of the current cell $\Omega_{P}$, subindex $f$ denotes the cell-faces, $\mathbf{A}_{f}=\left\|\mathbf{A}_{f}\right\| \hat{\mathbf{e}}_{f}$ is the area vector, $\hat{\mathbf{e}}_{f}$ is the unit vector orthogonal to the face $f$ pointing outside the current cell, as illustrated in Fig. 19a. Consistently with Eqs. (1, 12,5, 6): $(\beta, \mathbf{c}, \psi)=\left\{(1, \mathbf{v}, C),\left(\rho, \mathbf{v}, v_{j}\right),\left(1, \mathbf{v}, \phi_{i}\right),\left(1, \mathbf{n}_{i}^{0}, \phi_{i}\left(1-\phi_{i}\right)\right)\right\}, \quad v_{j}$ are the components of $\mathbf{v}, \mathbf{n}_{i}^{0}=\left.\mathbf{n}_{i}\right|_{\tau=0}$ (see reinitialization equation, Eq. (6)) $[10,49]$. If $\mathbf{c}_{f}=\mathbf{v}_{f}$ in Eq. (56), then $\mathbf{v}_{f}$ is given by Eq. (21). Whereas if $\mathbf{c}_{f}=\left(\mathbf{n}_{i}^{0}\right)_{f}$ in Eq. (56), then $\left(\mathbf{n}_{i}^{0}\right)_{f}$ is calculated by linear interpolation.

Unstructured flux-limiters, including Total variation diminishing (TVD) differencing schemes, are used for computing $\psi$ at the cell-faces, as first introduced in Balcázar et al. [10]. Fig. 19b shows a stencil of three points $\left\{\mathbf{x}_{C_{p}}, \mathbf{x}_{D_{p}}, \mathbf{x}_{U_{p}}\right\}$ on the line $l_{f}$, which is orthogonal to the face $f$ on the face centroid $\mathbf{x}_{f}$. Point $\mathbf{x}_{C}$ denotes the upwind cell centroid and $\mathbf{x}_{D}$ is the downwind cell centroid respect to the face $f$. The point $\mathbf{x}_{U}$ is the far-upwind cell-centroid, defined as the cell-centroid with the minimum distance to the line $l_{f}$ with $\mathbf{x}_{U_{p}}=\mathbf{x}_{C_{p}}-\Delta \mathbf{x}_{C_{p} \rightarrow D_{p}} \in l_{f}$. The far-upwind cell-centroid is selected from the set of cell-centroids around the vertices of the cell $\Omega_{C}$ (see Cells-layer 1 in Fig. 3c) in upwind direction with
1160

\section{6}

1157 
(a)



(b)

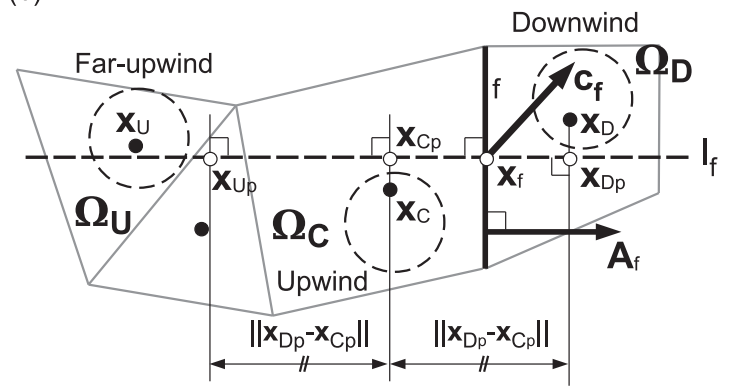


flux limiter schemes, $\mathbf{c}_{f} \in\left\{\mathbf{v}_{f}, \mathbf{n}_{f}^{0}\right\}$.

respect to the face $f$, as illustrated in Fig. 19b. Indeed, $\psi_{f}$ is written as the sum of a diffusive upwind part $\left(\psi_{C_{p}}\right)$ plus an anti-diffusive term [10,49]:

$\psi_{f}=\psi_{C_{p}}+\frac{1}{2} L\left(\theta_{f}\right)\left(\psi_{D_{p}}-\psi_{C_{p}}\right)$

The anti-diffusive part is multiplied by the flux limiter, $L\left(\theta_{f}\right)$, with $\theta_{f}$ defined as the upwind ratio of consecutive gradients of $\psi$, as follows: $\theta_{f}=\left(\psi_{C_{p}}-\psi_{U_{p}}\right) /\left(\psi_{D_{p}}-\psi_{C_{p}}\right)$. The flux limiters used in this work have the forms [78,79]:

$L\left(\theta_{f}\right) \equiv \begin{cases}\max \left\{0, \min \left\{2 \theta_{f}, 1\right\}, \min \left\{2, \theta_{f}\right\}\right\} & \text { TVD Superbee, } \\ \left(\theta_{f}+\left|\theta_{f}\right|\right) /\left(1+\left|\theta_{f}\right|\right) & \text { TVD Van - Leer, } \\ \max \left\{0, \min \left\{2 \theta_{f}, 0.25+0.75 \theta_{f}, 4\right\}\right\} & \text { Smart, } \\ 1 & \text { Central difference } \\ 0 & \text { Upwind. }\end{cases}$

A first-order Taylor approximation is used for computing $\psi$ at the points of the nodes-stencil $\left(k_{p}\right)$ :

$\psi_{k_{p}}=\psi_{k}+\left(\mathbf{x}_{k_{p}}-\mathbf{x}_{k}\right) \cdot(\nabla \psi)_{k}$,

where $\left(k_{p}, k\right)=\left\{\left(C_{p}, C\right),\left(D_{p}, D\right),\left(U_{p}, U\right)\right\}$ as illustrated in Fig. 19a. Further formulations of TVD differencing schemes can be found in [78,80-83].
Alternatively, $\psi=\phi(1-\phi)$, in the compressive term of the reinitialization equation (Eq. (6)), can be calculated at cell-faces by linear interpolation:

$\psi_{f}=w_{P} \psi_{P}+\left(1-w_{P}\right) \psi_{F}$.

Here, subindex $\{P, F\}$ denotes the cells with common face $f$, and $w_{P}$ is the interpolation coefficient. For instance, the distance weighted coefficient $w_{P}=1-\left\|\Delta \mathbf{x}_{P \rightarrow f}\right\|\left(\left\|\Delta \mathbf{x}_{P \rightarrow f}\right\|+\left\|\Delta \mathbf{x}_{F \rightarrow f}\right\|\right)^{-1}$ is used in this work unless otherwise stated; whereas other possibilities such as $w_{P}=0.5$ are incorporated in the computer-code. Eqs. (60) and (56) are used in this research to discretize the compressive term of Eq. (6).

Fig. 20 illustrates an assessment of the unstructured fluxlimiters schemes on the solution of the so-called Zalesak disc test [84]. The disc illustrated in Fig. 20a rotates in a circular domain of radius $R_{\Omega}=0.5$ and centroid $\mathbf{x}_{0}=\left(x_{0}, y_{0}\right)=(0.5,0.5)$. The rotation velocity is given by $\hat{\mathbf{e}}_{x} \cdot \mathbf{v}=y-y_{0}$ and $\hat{\mathbf{e}}_{y} \cdot \mathbf{v}=x_{0}-x$. The domain is discretized using $1.12 \times 10^{5}$ triangular cells, equivalent to the grid size $h \approx 2 R_{\Omega} / 250$. The CFL condition (see Eq. (16)) for time-step is set to $C_{t}=0.5$. As initial condition the marker function $\phi$ (Eq. (5)) is set to 0 inside the disc and 1 outside the disk, whereas Neumann boundary-condition is applied for all variables. Fig. 20bh show numerical solutions of the contours $\phi=\{0.05,0.5,0.95\}$, after one rotation of the disk around $\mathbf{x}_{0}$, as well as the exact solution. Fig. 20b-f depict results for upwind, van-Leer, and Superbee
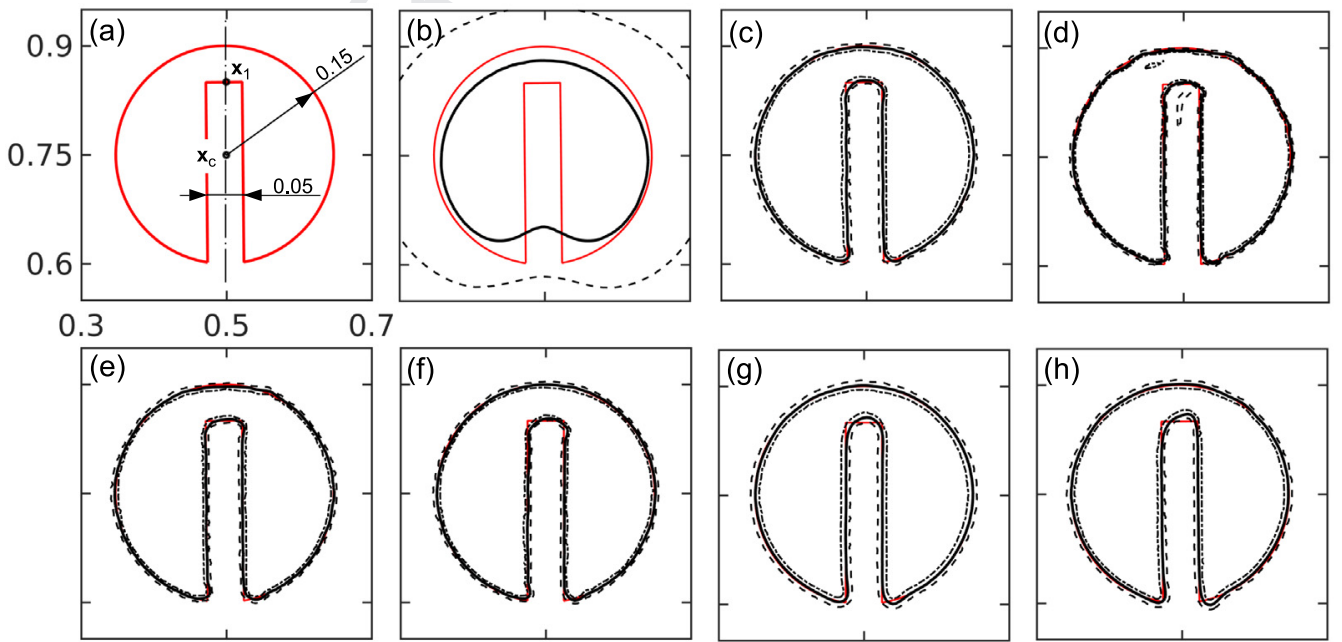



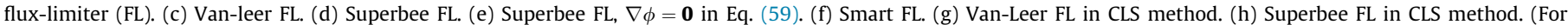
interpretation of the references to colour in this figure legend, the reader is referred to the web version of this article.) 



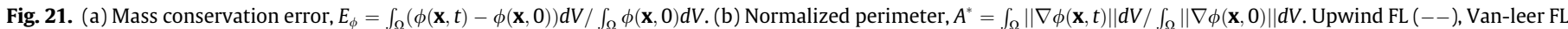
$(-\cdot)$, Superbee FL (.), Superbee FL with $\nabla \phi=\mathbf{0}$ in Eq. (59) (-), Smart FL (-, gray), Van-Leer FL in CLS method (--, gray), Superbee FL in CLS method (-., gray).

Table 4

Diffusive schemes. Terms of Eq. (62), where $\eta_{f}$ is the non-orthogonal contribution.

\begin{tabular}{lll}
\hline Diffusive scheme & $d_{f}$ & $\eta_{f}$ \\
\hline$D S 1$ & $\left\|\Delta \mathbf{x}_{P \rightarrow F}\right\|$ & $(\nabla \psi)_{f}^{i n t p} \cdot\left(\hat{\mathbf{e}}_{f}-\hat{\mathbf{e}}_{P \rightarrow F}\right)$ \\
$D S 2$ & $\left\|\Delta \mathbf{x}_{P_{p} \rightarrow F_{p}}\right\|$ & $\frac{\Delta \mathbf{x}_{F \rightarrow F_{p}} \cdot(\nabla \psi)_{F}-\Delta \mathbf{x}_{p \rightarrow P_{p}} \cdot(\nabla \psi)_{p}}{\left\|\Delta \mathbf{x}_{P_{p} \rightarrow F_{p}}\right\|}$ \\
\hline
\end{tabular}

flux-limiters, on the solution of advection equation (Eq. (5)); whereas Fig. 20g-h uses van-Leer and Superbee flux-limiters to solve the advection equation in the level-set method (Eqs. (5), (6)). As expected upwind flux-limiter is prone to excessive numerical diffusion, whereas other flux-limiters present an excellent performance. Results with Superbee flux-limiter (see Fig. 20d) tend to oscillate, because the thickness $(0.05 \leqslant \phi \leqslant 0.95)$ of the disk is extremely sharp, whereas the accuracy of Eq. (59) is affected by the gradients. As depicted in Fig. 20e, the last issue is solved by deactivating the gradient correction in Eq. (59). On the other hand, as illustrated in Fig. 20h, Superbee flux-limiter is a reliable scheme in the context of the CLS method, since the thickness of the CLS profile $(0.05 \leqslant \phi \leqslant 0.95)$ can be smoothed by solving the reinitialization equation (Eq. (6)). Fig. 21 depict the time evolution of mass conservation error $\left(E_{\phi}\right)$ and normalized perimeter of the $\operatorname{disc}\left(A^{*}\right)$. It is observed excellent mass conservation for all flux-limiters $\left(10^{-16}<E_{\phi}<10^{-11}\right)$, whereas upwind flux-limiter achieves a maximum error $E_{\phi}<10^{-8}$. Since excessive numerical diffusion, the disk shape is not well preserved by upwind limiter, whereas it is well preserved by the other flux-limiters (see $A^{*}(t)$ in Fig. 21).

\section{A.2. Diffusive schemes}

Diffusive term is discretized at the cell $\Omega_{P}$ as follows [10]:

$\left(\nabla_{h} \cdot \zeta \nabla_{h} \psi\right)_{P} \approx \frac{1}{V_{P}} \sum_{f} \zeta_{f}\left(\nabla_{h} \psi\right)_{f} \cdot \mathbf{A}_{f}$.

A central difference scheme with non-orthogonal correction $[85,86]$ is used for computing diffusive fluxes at cell-faces: $\left(\nabla_{h} \psi\right)_{f} \cdot \mathbf{A}_{f}=\left\|\mathbf{A}_{f}\right\|\left(\frac{\psi_{F}-\psi_{P}}{d_{f}}+\eta_{f}\right)$

where $d_{f}$ and $\eta_{f}$ (non-orthogonal contribution) are defined in Table 4. Here, $\Delta \mathbf{x}_{i \rightarrow j}=\mathbf{x}_{j}-\mathbf{x}_{i}, \hat{\mathbf{e}}_{P \rightarrow F}=\left(\left\|\Delta \mathbf{x}_{P \rightarrow F}\right\|\right)^{-1} \Delta \mathbf{x}_{P \rightarrow F}, \hat{\mathbf{e}}_{f}$ is the unit vector orthogonal to the face $f$ pointing outside the cell $P$, as illustrated in Fig. 19a. Consistently with Eqs. (12, 1,6): $\left(\zeta_{f}, \psi\right)=\left\{\left(\mathcal{D}_{c . f}, C\right),\left(\mu_{f}, v_{j}\right),\left(\varepsilon_{f}, \phi_{i}\right)\right\}$, where $\zeta_{f}$ and gradients at cell-faces are computed by linear interpolation, e.g., $(\nabla \psi)_{f}^{\text {intp }}=w_{P}\left(\nabla_{h} \psi\right)_{P}+\left(1-w_{P}\right)\left(\nabla_{h} \psi\right)_{F}$. While other diffusive schemes (see Jasak [86]) can be defined in the framework of Eq. (62), results of numerical experiments performed in this work (see Fig. 7)) are not sensitive to the selected scheme (Table 4). As a consequence, diffusive scheme DS1 (see Table 4) is used unless otherwise stated.

\section{Appendix B. Parallel scalability}

The numerical methods used in this work are implemented in the framework of an in-house $\mathrm{C}++$ computer code called TermoFluids [60], which employs the Message Passing Interface (MPI) for parallel communications. The parallel scalability of these methods and computer code were researched simulating the buoyancy-driven motion of a swarm of 24 bubbles, including mass transfer and first-order chemical reaction, using $E o=3.125$, $M o=5 \times 10^{-6}, \eta_{\rho}=100, S c=1$ and $\eta_{\mu}=100 . \Omega$ is a cylindrical pipe, with size $\left(D_{\Omega}, H_{\Omega}\right)=\left(6 d_{b}, 4 d_{b}\right)$, where $D_{\Omega}$ is the cylinder diameter, $H_{\Omega}$ is the cylinder height, and $d_{b}$ is the bubble diameter. $\Omega$ is divided in $24.9 \times 10^{6}$ triangular prisms cells, which corresponds to the grid size $h=d_{b} / 40$. These test cases were carried-out on the supercomputer MareNostrum IV based in Barcelona-Spain. The scalability of the computer code was investigated by using 480 up to 4032 cores. Fig. 22a depicts the normalized time required for one-iteration of the global algorithm, using the computing time with 480 processors as the reference time $\left(T_{\text {ref }}\right)$. Furthermore, Fig. 22b presents the strong speed-up (SSU) computed as $S S U=T_{\text {ref }} / T_{\text {comp }}$, where $T_{\text {comp }}$ is the computing time per iteration of the global algorithm.
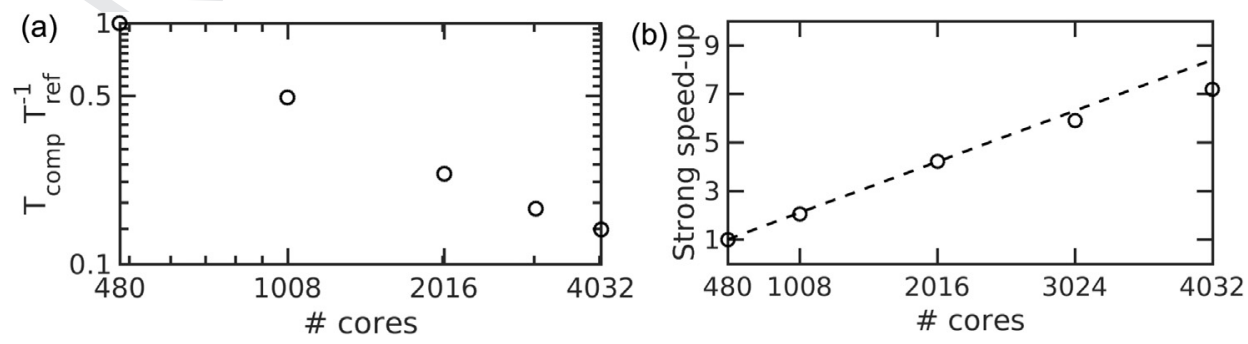


linear (- ) and present results (०). 


\section{References}

[1] R. Mudde, Gravity-driven bubbly flows, Annu. Rev. Fluid Mech. 37 (2005) 393 423.

[2] P.A. Ramachandran, R.V. Chaudhari, Three Phase Catalytic Reactors, Gordon and Breach, New York, 1983.

[3] P.L. Mills, R.V. Chaudhari, Catal. Today 48 (1999) 17

[4] X. Chen, Z. Xu, P. Cen, W.K.R. Wong, Process Biochem., Oxford, U.K., 40, 1 (2005).

[5] R. Clift, J.R. Grace, M.E. Weber, Bubbles, Drops and Particles, Academic Press, New York, 1978

[6] G. Tryggvason, S. Dabiri, B. Abouhasanzadeh, L. Jaicai, Multiscale considerations in direct numerical simulations of multiphase flows, Phys. Fluids 25 (2013), 031302

[7] S. Osher, J.A. Sethian, Fronts propagating with curvature-dependent speed: algorithms based on Hamilton-Jacobi formulations, J. Comput. Phys. 79 (1988) $175-210$.

[8] M. Sussman, P. Smereka, S. Osher, A level set approach for computing solutions to incompressible two-phase flow, J. Comput. Phys. 144 (1994) 146-159.

[9] E. Olsson, G. Kreiss, A conservative level set method for two phase flow, J. Comput. Phys. 210 (2005) 225-246.

[10] N. Balcázar, L. Jofre, O. Lehmkhul, J. Castro, J. Rigola, A finite-volume/level-set method for simulating two-phase flows on unstructured grids, Int. J. Multiph. Flow 64 (2014) 55-72.

[11] C. Hirt, B. Nichols, Volume of fluid (VOF) method for the dynamics of free boundary, J. Comput. Phys. 39 (1981) 201-225.

[12] R. Scardovelli, S. Zaleski, Direct numerical simulation of free surface and interfacial flow, Annu. Rev. Fluid Mech. 31 (1999) 567-603.

[13] M. Sussman, E.G. Puckett, A coupled level set and volume-of-fluid method for computing 3D and axisymmetric incompressible two-phase flows, J. Comput. Phys. 162 (2000) 301-337.

[14] D.L. Sun, W.Q. Tao, A coupled volume-of-fluid and level-set (VOSET) method for computing incompressible two-phase flows, Int. J. Heat Mass Transf. 53 (2010) 645-655.

[15] I. Chakraborty, G. Biswas, P.S. Ghoshdastidar, A coupled level-set and volumeof-fluid method for the buoyant rise of gas bubbles in liquids, Int. J. Heat Mass Transf. 58 (2013) 240-259.

[16] N. Balcázar, O. Lehmkhul, L. Jofre, J. Rigola, A. Oliva, A coupled volume-of-fluid/ level-set method for simulation of two-phase flows on unstructured meshes, Comput. Fluids 124 (2016) 12-29.

[17] G. Tryggvason, B. Bunner, A. Esmaeeli, D. Juric, N. Al-Rawahi, W. Tauber, J. Han, S. Nas, Y.-J. Jan, A front-tracking method for the computations of multiphase flow, J. Comput. Phys. 169 (2001) 708-759.

[18] M.R. Davidson, M. Rudman, Volume-of-fluid calculation of heat or mass transfer across deforming interfaces in two-fluid flow, Numer. Heat Transf., Part B: Fundam. 41 (2002) 291-308.

[19] D. Bothe, M. Koebe, K. Wielage, H.J. Warnecke, VoF simulations of mass transfer from single bubbles and bubble chains rising in the aqueous solutions, in: Proceedings of FEDSM03: Fourth ASME-JSME Joint Fluids Engineering Conference, Honolulu, HI, USA, July 6-11, 2003.

[20] A. Alke, D. Bothe, M. Kroeger, H.-J. Warnecke, VOF-based simulation of conjugate mass transfer from freely moving fluid particles, in: A.A. Mammoli, C.A. Brebbia (Eds.) Computational Methods in Multiphase Flow V, WIT Transactions on Engineering Sciences, 2009, pp. 157-168.

[21] A. Onea, M. Wörner, D.G. Cacuci, A qualitative computational study of mass transfer in upward bubble train flow through square and rectangular minichannels, Chem. Eng. Sci. 64 (2009) 1416-1435.

[22] R. Gupta, D.F. Fletcher, B.S. Haynes, CFD modelling of flow and heat transfer in the Taylor flow regime, Chem. Eng. Sci. 65 (2010) 2094-2107.

[23] K. Hayashi, A. Tomiyama, Interface tracking simulation of mass transfer from a dissolving bubble, J. Comput. Multiphase Flows 3 (2011) 247-262.

[24] D. Bothe, M. Koebe, K. Wielage, J. Pruss, H.-J. Warnecke, Direct Numerical Simulation of Mass Transfer Between Rising Gas Bubbles and Water, Sommerfeld M. ed., Analysis, Modelling and Calculation, Bubble Flows, 2004, pp. 159-174.

[25] D. Bothe, M. Kröger, H.J. Warnecke, A VOF-based conservative method for the simulation of reactive mass transfer from rising bubbles, Fluid Dynam. Mater. Process. 7 (2011) 303-316.

[26] M. Francois, N. Carlson, The global embedded interface formulation for interfacial mass transfer within a volume tracking framework, Comput. Fluids 87 (2013) 102-114.

[27] D. Bothe, S. Fleckenstein, Modeling and VOF-based numerical simulation of mass transfer processes at fluidic particles, Chem. Eng. Sci. 101 (2013) 283302.

[28] A. Weiner, D. Bothe, Advanced subgrid-scale modeling for convectiondominated species transport at fluid interfaces with application to mass transfer from rising bubbles, J. Comput. Phys. 347 (2017) 261-289.

[29] H. Marshall, K. Hinterberger, C. Schüler, F. Habla, O. Hinrichsen, Numerical simulation of species transfer across fluid interfaces in free-surface flows using OpenFOAM, Chem. Eng. Sci. 78 (2012) 111-127.

[30] D. Deising, H. Marschall, D. Bothe, A unified single-field model framework for volume-of-fluid simulations of interfacial species transfer applied to bubbly flows, Chem. Eng. Sci. 139 (2016) 173-195.

[31] C. Yang, Z.S. Mao, Numerical simulation of interphase mass transfer with the level set approach, Chem. Eng. Sci. 60 (2005) 2643-2660.
[32] J. Wang, P. Lu, Z. Wang, C. Yang, Z.S. Mao, Numerical simulation of unsteady mass transfer by the level set method, Chem. Eng. Sci. 63 (2008) 3141-3151.

[33] A.A. Ganguli, E.Y. Kenig, A CFD-based approach to the interfacial mass transfer at free gas-liquid interfaces, Chem. Eng. Sci. 66 (2011) 3301-3308.

[34] D. Darmana, N.G. Deen, J.A.M. Kuipers, Detailed 3D modeling of mass transfer processes in two-phase flows with dynamic interfaces, Chem. Eng. Technol. 29 (9) (2006) 1027-1033.

[35] P. Bhuvankar, S. Dabiri, Impact of a single bubble rising near a wall on the wallto-liquid heat flux, Int. J. Heat Mass Transf. 116 (2018) 445-457.

[36] Z.S. Mao, T. Li, J. Chen, Numerical simulation of steady and transient mass transfer to a single drop dominated by external resistance, Int. J. Heat Mass Transf. 44 (2001) 1235-1247.

[37] B. Figueroa-Espinoza, D. Legendre, Mass or heat transfer from spheroidal gas bubbles rising through a stationary liquid, Chem. Eng. Sci. 65 (2010) 62966309.

[38] M. Falcone, D. Bothe, H. Marschall, 3D direct numerical simulations of reactive mass transfer from deformable single bubbles: an analysis of mass transfer coefficients and reaction selectivities, Chem. Eng. Sci. 177 (2018) 523-536.

[39] S. Dabiri, G. Tryggvason, Heat transfer in turbulent bubbly flow in vertical channels, Chem. Eng. Sci. 122 (2015) 106-113.

[40] S. Piedra, J. Lu, E. Ramos, G. Tryggvason, Numerical study of the flow and heat transfer of bubbly flows in inclined channels, Int. J. Heat Fluid Flow 56 (2015) 43-50.

[41] N.G. Deen, J.A.M. Kuipers, Direct numerical simulation of wall to liquid heat transfer in dispersed gas-liquid two-phase flow using a volume of fluid approach, Chem. Eng. Sci. 102 (2013) 268-282.

[42] B. Aboulhasanzadeh, S. Thomas, M. Taeibi-Rahni, G. Tryggvason, Multiscale computations of mass transfer from buoyant bubbles, Chem. Eng. Sci. 75 (2012) 456-467.

[43] B. Aboulhasanzadeh, S. Hosoda, A. Tomiyama, G. Tryggvason, A validation of an embedded analytical description approach for the computations of high schmidt number mass transfer from bubbles in liquids, Chem. Eng. Sci. 101 (2013) 165-174.

[44] B. Aboulhasanzadeh, G. Tryggvason, Effect of bubble interactions on mass transfer in bubbly flow, Int. J. Heat Mass Transf. 79 (2014) 390-396.

[45] I. Roghair, M. Van Sint Annaland, J.A.M. Kuipers, An improved front-tracking technique for the simulation of mass transfer in dense bubbly flows, Chem. Eng. Sci. 152 (2016) 351-369.

[46] A. Koynov, J.G. Khinast, G. Tryggvason, Mass transfer and chemical reactions in bubble swarms with dynamic interfaces, AIChE J. 51 (10) (2005) 2786-2800.

[47] S. Radl, A. Koynov, G. Tryggvason, J. Khinast, DNS-based prediction of the selectivity of fast multiphase reactions: hydrogenation of nitroarenes, Chem. Eng. Sci. 63 (2008) 3279-3291.

[48] N. Balcázar, O. Lehmkhul, J. Rigola, A. Oliva, A multiple marker level-set method for simulation of deformable fluid particles, Int. J. Multiph. Flow 74 (2015) 125-142.

[49] N. Balcázar, J. Rigola, J. Castro, A. Oliva, A level-set model for thermocapillary motion of deformable fluid particles, Int. J. Heat Fluid Flow 62 (Part B) (2016) 324-343.

[50] O. Antepara, O. Lehmkhul, R. Borrell, J. Chiva, A. Oliva, Parallel adaptive mesh refinement for large-eddy simulations of turbulent flows, Comput. Fluids 110 (2015) 48-61.

[51] E. Coyajee, B.J. Boersma, Numerical simulation of drop impact on a liquidliquid interface with a multiple marker front-capturing method, J. Comput. Phys. 228 (12) (2009) 4444-4467.

[52] A. Esmaeeli, G. Tryggvason, Direct numerical simulations of bubbly flows Part 2. Moderate Reynolds number arrays, J. Fluid Mech. 385 (1999) 325-358.

[53] N. Balcázar, J. Castro, J. Rigola, A. Oliva, DNS of the wall effect on the motion of bubble swarms, Proc. Comput. Sci. 108 (2017) 2008-2017.

[54] D.J. Mavriplis, Unstructured Mesh Discretizations and Solvers for Computational Aerodynamics, in: 18th Computational Fluid Dynamics Conference, AIAA Paper 2007-3955, Miami, FL, 2007, https://doi.org/10.2514/ 6.2007-3955.

[55] J.U. Brackbill, D.B. Kothe, C. Zemach, A continuum method for modeling surface tension, J. Comput. Phys. 100 (1992) 335-354.

[56] B. Engquist, A.K. Tornberg, R. Tsai, Discretization of Dirac delta functions in level set methods, J. Comput. Phys. 207 (2005) 28-51.

[57] A.J. Chorin, Numerical solution of the Navier-Stokes equations, Math. Comput. 22 (1968) 745-762.

[58] S. Gottlieb, C.W. Shu, Total variation dimishing Runge-Kutta schemes, Math. Comput. 67 (1998) 73-85.

[59] C.M. Rhie, W.L. Chow, Numerical study of the turbulent flow past an airfoil with trailing edge separation, AIAA J. 21 (1983) 1525-1532.

[60] Termo Fluids S.L., web page: <http://www.termofluids.com/>.

[61] K. Schloegel, G. Karypis, V. Kumar, ParMETIS, parallel graph partitioning and sparse matrix ordering library, J. Comput. Phys. (2009).

[62] N. Balcázar, J. Castro, J. Chiva, A. Oliva, DNS of falling droplets in a vertical channel, Int. J. Comput. Methods Exp. Meas. 6 (2018) 398-410, https://doi.org/ 10.2495/CMEM-V6-N2-398-410.

[63] N. Balcázar, O. Lemhkuhl, L. Jofre, A. Oliva, Level-set simulations of buoyancydriven motion of single and multiple bubbles, Int. J. Heat Fluid Flow 56 (2015) 91-107.

[64] D. Colombet, D. Legendre, F. Risso, A. Cockx, P. Guiraud, Dynamics and mass transfer of rising bubbles in a homogenous swarm at large gas volume fraction, J. Fluid Mech. 763 (2015) 254-285.

[65] J. Crank, The Mathematics of Diffusion, 2nd ed., Clarendon Press, Oxford, 1975.

1380 
[66] G. Batchelor, Small-scale variation of convected quantities like temperature in turbulent fluid Part 1. General discussion and the case of small conductivity, J. Fluid Mech. 5 (1959) 113-133.

[67] R.F. Mudde, D.J. Lee, J. Reese, L. Fan, Role of coherent structures on Reynolds stresses in a 2D bubble column, AIChE J. 43 (1997) 913-926.

[68] W. Dijkhuizen, I. Roghair, M. Van Sint Annaland, J.A.M. Kuipers, DNS of gas bubbles behaviour using an improved 3D front tracking modelDrag force on isolated bubbles and comparison with experiments, Chem. Eng. Sci. 65 (2010) 1415-1426.

[69] M. Rastello, J. Marie, M. Lance, Drag and lift forces on clean spherical and ellipsoidal bubbles in a solid-body rotating flow, J. Fluid Mech. 682 (2011) 434-459.

[70] J. Boussinesq, Calcul du pouvoir refroidissant des courants fluides, J. Math. Pures Appl. 6 (1905) 285-332.

[71] S. Winnikow, Letter to the editors, Chem. Eng. Sci. 22 (3) (1967) 477.

[72] F. Takemura, A. Yabe, Gas dissolution process of spherical rising gas bubbles, Chem. Eng. Sci. 53 (15) (1998) 2691-2699.

[73] A. Lochiel, P. Calderbank, Mass transfer in the continuous phase around axisymmetric bodies of revolution, Chem. Eng. Sci. 19 (1964) 471-484.

[74] I. Roghair, Y.M. Lau, N.G. Deen, H.M. Slagter, M.W. Baltussen, M. Van Sint Annaland, J.A.M. Kuipers, On the drag force of bubbles in bubble swarms at intermediate and high Reynolds numbers, Chem. Eng. Sci. 66 (2011) 32043211.

[75] A. Bridge, L. Lapidus, J. Elgin, The mechanics of vertical gas-liquid fluidized system I: countercurrent flow, AIChE J. 10 (6) (1964) 819-826.

[76] M. Simonnet, C. Gentric, E. Olmos, N. Midoux, Experimental determination of the drag coefficient in a swarm of bubbles, Chem. Eng. Sci. 62 (3) (2007) 858 866.
[77] H. Rusche, R. Issa, The effect of voidage on the drag force on particles, droplets and bubbles in dispersed two-phase flow, in: Japanese European Two-Phase Flow Meeting, Tsukuba, Japan, 2000.

[78] P.K. Sweby, High resolution using flux limiters for hyperbolic conservation laws, SIAM J. Numer. Anal. 21 (1984) 995-1011.

[79] P.H. Gaskell, A.K.C. Lau, Curvature-compensated convective transport: SMART a new boundedness-preserving transport algorithm, Int. J. Numer. Methods 8 (1988) 617-641.

[80] M.S. Darwish, F. Moukalled, TVD schemes for unstructured grids, Int. J. Heat Mass Transf. 46 (2003) 599-611.

[81] L. Lian-xia, L. Hua-sheng, Q. Li-jian, An improved r-factor algorithm for TVD schemes, Int. J. Heat Mass Transf. 51 (2008) 610-617.

[82] J. Hou, F. Simons, R. Hinkelmann, Improved total variation diminishing schemes for advection simulation on arbitrary grids, Int. J. Numer. Meth Fluids 70 (2012) 359-382.

[83] F. Denner, B. van Wachem, TVD differencing on three-dimensional unstructured meshes with monotonicity-preserving correction of mesh skewness, J. Comput. Phys. 298 (2015) 466-479.

[84] S. Zalesak, Fully multidimensional flux-corrected transport algorithms for fluids, J. Comput. Phys. 31 (1979) 335-362.

[85] C.D. Perez-Segarra, C. Farre, J. Cadafalch, A. Oliva, Analysis of different numerical schemes for the resolution of convection-diffusion equations using finite-volume methods on three-dimensional unstructured grids. Part I: discretization schemes, Numer. Heat Transf. B 49 (2006) 333-350.

[86] H. Jasak, Error analysis and estimation in the Finite Volume method with applications to fluid flows, Ph.D. Thesis, Imperial College, University of London, 1996. 